\title{
Choice History Bias in Intertemporal Choice
}

A PREPRint, MANUSCRIPT VERSION 2.0, 10/27/2020

\author{
Martin Schoemann * \\ Department of Psychology \\ Technische Universität Dresden \\ 01062 Dresden, Germany \\ martin.schoemann@tu-dresden.de
}

\author{
Stefan Scherbaum \\ Department of Psychology \\ Technische Universität Dresden \\ 01062 Dresden, Germany \\ stefan.scherbaum@tu-dresden.de
}

\begin{abstract}
Human decision making is prone to many biases that either result from properties of the actual decision or from properties of the decision environment. We investigated the influence of the choice history on the actual decision in the domain of intertemporal choice, known as choice history bias from perceptual decision making. Over a series of three experiments, we demonstrate that the choice history bias also operates in intertemporal choice, but only under specific circumstances. We identified the inter-trial interval to be a determinant of the bias. Our results corroborate recent findings investigating path-dependence of perceptual and preferential decisions, and consolidate the overall mechanistic interpretation that the choice history bias arises due to residual activity in the neural system. Hence, our study bears two implications: First, models of intertemporal choice need to consider the dependency of choices across trials; second, the study of intertemporal choices empirically asks for considering this path-dependence to avoid biased conclusions about individual choices.
\end{abstract}

Keywords Choice repetition · Choice history $\cdot$ Mouse cursor tracking $\cdot$ Process dynamics $\cdot$ Hysteresis

\section{Introduction}

Decisions are often shaped by cognitive biases (Erev et al., 2017; Tversky \& Kahneman, 1974). Over six decades of psychological research in human judgment and decision making has produced an impressive list of such cognitive biases (Baron, 2008, see his Table 2.1) though a unifying, process-oriented framework that explains their causal mechanisms is still missing (Hilbert, 2012). For some of those biases, recent process-oriented research provided evidence for a link between neural and choice variability (Bogdanov et al., 2015; Haller \& Schwabe, 2014; Nicolle et al., 2011) suggesting that choice biases can emerge due to basal properties of the neural system (Gold \& Shadlen, 2007; Hunt, 2014). Here, we incorporate this line of research with regard to choice history biases - the tendency to stick to the previously chosen option against the odds from the normative perspective. We demonstrate the occurrence of the phenomenon and its boundary conditions in the domain of intertemporal choice, and argue that choice history biases might arise due to residual activation of the previously chosen option.

Choice repetition biases and more complex sequential effects are well-established phenomena in the perceptual domain in simple two-alternative forced-choice (2AFC) reaction-time tasks since the 1960's (Bertelson, 1965; Hale, 1967; N. H. Kirby, 1976; Perea \& Carreiras, 2003; Quinlan, 1999; Remington, 1969; Soetens et al., 2004; Soetens, 1998; Soetens et al., 1985; Vervaeck \& Boer, 1980). From a mechanistic point of view, such effects can be captured by computational modeling of biophysical competitive attractor networks, proposing a direct link between neural and choice/reaction time variability (e.g., Berlemont \& Nadal, 2019; Cho et al., 2002; Gao et al., 2009). This notion

\footnotetext{
${ }^{*}$ Corresponding author; Department of Management, Aarhus University, 8000 Aarhus, Denmark, ms@mgmt .au .dk; Primary data and analysis scripts to reproduce all results and figures of the article are available via osf.io/rbxv5/; the preregistration of the third experiment is available via osf.io/57qpm/. We report achieved power for the used samples, all data exclusions (if any), and all relevant measures and manipulations in the study.
} 
is supported by another, closely related line of research eliciting and exploring the impact of hysteresis ${ }^{2}$ on human performance in numerous psychophysical tasks such as motion perception (e.g., Hock et al., 2003), speech perception (e.g., Tuller et al., 1994), and objects recognition (e.g., Kleinschmidt et al., 2002). In this line of research too, dynamic modeling via competitive attractor networks proved very successful and proposed a link between neural and perceptual variability (Case et al., 1995; Kelso, 1997; Kelso et al., 1995).

More recently, the proposed link between neural and choice variability has been decomposed by combining predictions from computational modeling of competitive attractor networks with experimental findings from non-invasive brain stimulation (Bonaiuto et al., 2016; Hämmerer et al., 2016). The work of Bonaiuto et al. (2016) and Hämmerer et al. (2016) showed that biophysical competitive attractor networks generate perceptual decision making behavior accurately matching that of human participants (see also Usher \& McClelland, 2001; Wang, 2008, 2012; Wong \& Wang, 2006; Wong et al., 2007), and that sustained (residual) recurrent activity in such networks is the basic mechanism behind choice repetition biases in perceptual decision making. From this basic mechanism, it becomes also evident that the gradually declining residual activity following a decision should bias the subsequent decision stronger in tasks with short inter-trial intervals (Bonaiuto et al., 2016; Hämmerer et al., 2016).

While choice history biases and hysteresis gained a lot of attention in perceptual and simple choice tasks (Abrahamyan et al., 2016), they have only been scarcely investigated in preferential decision making. This is particularly surprising since there is converging evidence that also for preferential decision making the link between neural and choice variability seems to be well explained by competitive attractor networks (Bogacz et al., 2007; Hunt et al., 2012; Jocham et al., 2012; Usher \& McClelland, 2004). For instance, van Rooij et al. (2013) investigated hysteresis in risky choice which was captured by a simple one-dimensional attractor model. Other studies from our own lab elicited and explored hysteresis in a gamified delay discounting task combined with computational modeling of an attractor model (Scherbaum et al., 2016; Schoemann \& Scherbaum, 2020). In some of our more recent work, we also validated the attractor model's specific prediction (as depicted in Figure 1) that the influence of the decision history on the current decision (i.e., choice repetition, hysteresis) negatively correlates with the duration of the inter-trial interval (Senftleben et al., 2020; Senftleben et al., 2019).
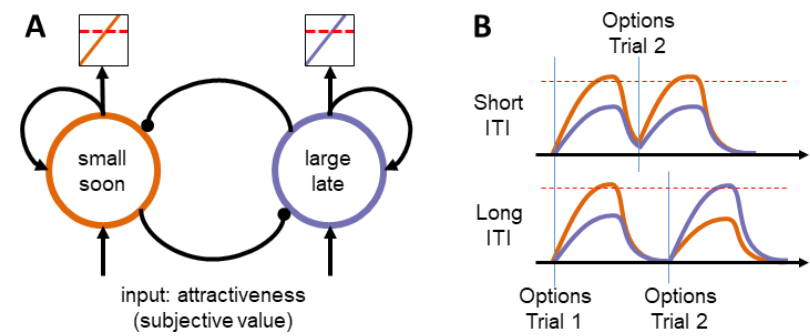

Figure 1: A: Simple neural attractor model of intertemporal choice, e.g., for a small but soon (SS) and a large but late (LL) options as previously used in gamified studies on the choice history bias in delay discounting. B: Prediction of choice history biased based on residual activation for a scenario, in which the SS (orange) option is more attractive in the first trial, but the LL (purple) option is slightly more attractive in the second trial. B upper graph: If only a short inter-trial interval separates the two trials, residual activation of the winning SS option (orange) supports a repetition of the choice. B lower graph: If a long inter-trial interval separates the two trials, residual activation is decayed so that a switch to the now more attractive LL option (purple) can occur.

With this study, we wanted to investigate whether our previous findings regarding choice history biases and the effect of the inter-trial interval (ITI) generalize to the classic intertemporal choice (ITC). ITC comprises decisions between a small but soon option ( $S S$, e.g., 5 Euros now) and a large but late option ( $L L$, e.g., 10 Euros in 14 days). In order to study choice history biases in ITC, we, first, developed a classic ITC paradigm that allows for eliciting and measuring choice repetition on a cognitive processing rather than a mere motor execution level (Experiment 1 and 2); second, we tested the prediction that choice repetition occurs more strongly in tasks with shorter ITIs (Experiment 3, preregistered).

\section{Experiment 1}

In our first experiment, we tested a novel version of a classic intertemporal choice (ITC) paradigm which had to fulfill the following two requirements: (1) being comparable to our previous work on ITC with regard to temporal discounting

\footnotetext{
${ }^{2}$ In the context of perception, hysteresis describes the persistence of an initial percept despite a change in stimulus properties which favors an alternative interpretation (Sekuler, 1996)
} 
and its action dynamics (Dshemuchadse et al., 2013; Scherbaum et al., 2018a, 2018b), and (2) eliciting choice history bias on a cognitive processing rather than a mere motor execution level akin to our previous work using the gamified delay discounting task (Scherbaum et al., 2016; Schoemann \& Scherbaum, 2020; Senftleben et al., 2020; Senftleben et al., 2019). The latter requirement reflects the fact, that in ITC tasks, options are usually presented on the same side throughout the whole experiment, for instance, the SS option on the left side and the LL option on the right side (e.g., Franco-Watkins et al., 2015). Hence, if a choice was repeated, one could not separate the mere tendency to repeat a previously performed motor command (e.g., left response) from the tendency to repeat a previously performed choice of a certain option (e.g., SS option).

To meet those requirements, we adapted our mouse-tracking based ITC task to varying response locations using the same range of values and delays as in Dshemuchadse et al. (2013). Furthermore, we used the same experimental block design as in our previous work (Schoemann \& Scherbaum, 2020; Senftleben et al., 2020) in such a way that a first block served to measure participants' temporal discounting which was then, in a second block, used to construct individualized series of choices in which the choice history bias was measured.

For the first block, we hypothesized that (H1) participants' choice behavior would show temporal discounting, as well as, that (H2) participants' action dynamics (i.e., mouse cursor trajectories) while making those choices would reflect more conflict when people choose the LL option compared to the SS option depending on the difficulty of the choices (Dshemuchadse et al., 2013; Scherbaum et al., 2013). For the second block, we hypothesized that (H3) participants' choice behavior would show a choice history bias (i.e., hysteresis) in such a way that in a sequence of choices where the initially unchosen option becomes increasingly more attractive, people switch to this now more attractive option much later than they would if their choices were unbiased by the choice history (Scherbaum et al., 2016; Schoemann \& Scherbaum, 2020; Senftleben et al., 2020; Senftleben et al., 2019). Furthermore, we expected that (H4) decision times should increase until people switch from choosing one option over the alternative option, and decrease after that switch (Frank et al., 2015; Senftleben et al., 2020; Senftleben et al., 2019).

\subsection{Method}

\subsubsection{Participants}

We recruited forty-nine participants $(77.55 \%$ female, mean age $=24.86$ years, range $=19-62)$ through the department's data-base system ORSEE (Greiner, 2015). The experiment was conducted at the Technische Universität Dresden and lasted approximately 45 minutes; participants gave informed consent and received either class credits or $€ 5.00$ show-up fee; all participants had normal or corrected-to-normal vision. According to the experimental protocol, twelve participants had to finish the experiment after the first block due to insufficient temporal discounting (see Procedure). Hence, we achieved a final sample size of thirty-seven participants $(78.37 \%$ female, mean age $=24.70$ years, range $=$ 19-49) for all subsequent analyses. A sensitivity analysis using G*Power (Faul et al., 2009) revealed that a sample size of $N>35$ is sufficient to detect a medium sized choice repetition effect, $d=0.50$ (see Schoemann \& Scherbaum, 2020), with a power of $1-\beta>.90$.

\subsubsection{Apparatus and stimuli}

The experiment was presented on a 17 -inch screen $(1280$ x 1024 pixels, $85 \mathrm{~Hz})$. As presentation software, we used Psychophysics Toolbox 3 (Brainard, 1997; Pelli, 1997) in Matlab 2010b (the Mathworks Inc.), running on a Windows XP SP2 personal computer. Responses were carried out by moving a high precision computer mouse (Logitech Laser Mouse USB). Mouse cursor trajectories were sampled with a frequency of $95 \mathrm{~Hz}$. Recording started at presentation of the choice options and lasted until participants indicated their choice by clicking into specified areas underlaying either option (see Procedure).

In ITC, participants are confronted with a choice between a soon-small (SS) and a late-large (LL) option. Hence, either option is described by two attributes, namely, reward and delay. Both attributes were represented in term of numbers using two digits, both to the left and right of the decimal point (see Figure 1). Rewards were identifiable due to a Euro sign ' $€$ ', whereas delays were identified by a following 'd' indicating days as the time unit (see Figure 1). The magnitude of rewards ranged from $€ 0.23$ to $€ 20.32$. The magnitude of delay ranged from 1 day to 21 days.

\subsubsection{Procedure}

Participants were asked to decide on each trial which of two options (SS vs LL) they preferred. Participants were instructed to respond to the hypothetical choices as if they were real choices. For reasons of practicability, we used hypothetical choices, as previous studies showed that they are behaviorally comparable to real choices (Johnson \& Bickel, 2002; Urminski \& Zauberman, 2014). Additionally, participants were instructed, that the experiment might 
finish after the first block due to their choice behavior. They were specifically told that they should treat the hypothetical choice as real choices, and that the experiment is able to detect if they did not (see Design). Each trial consisted of two stages: the start stage and the response stage. In the start stage, participants had to click on the fixation cross presented at the center of the screen (see Figure 2A). Along with the click, the cursor was positioned at the exact center of the screen. This procedure served to guarantee the same starting point of the cursor movement for each trial. With the click on the fixation cross the response stage started. In the response stage, the two choice options appeared on the screen, with the delay of each option presented below the reward. Underlaying either choice option, a circular response area was presented (see Figure 2). Participants had to click into either response area to indicate their choice. After participants indicated their response in the trial, a blank screen was presented for an inter-trial interval of 0.3 seconds. Between trials, we varied the position of the choice options, and hence, the response areas in such a way that they rotated clock-wise around the center of the screen, with the LL option always being next to the SS option in the direction of the rotation (see Figure 2B).
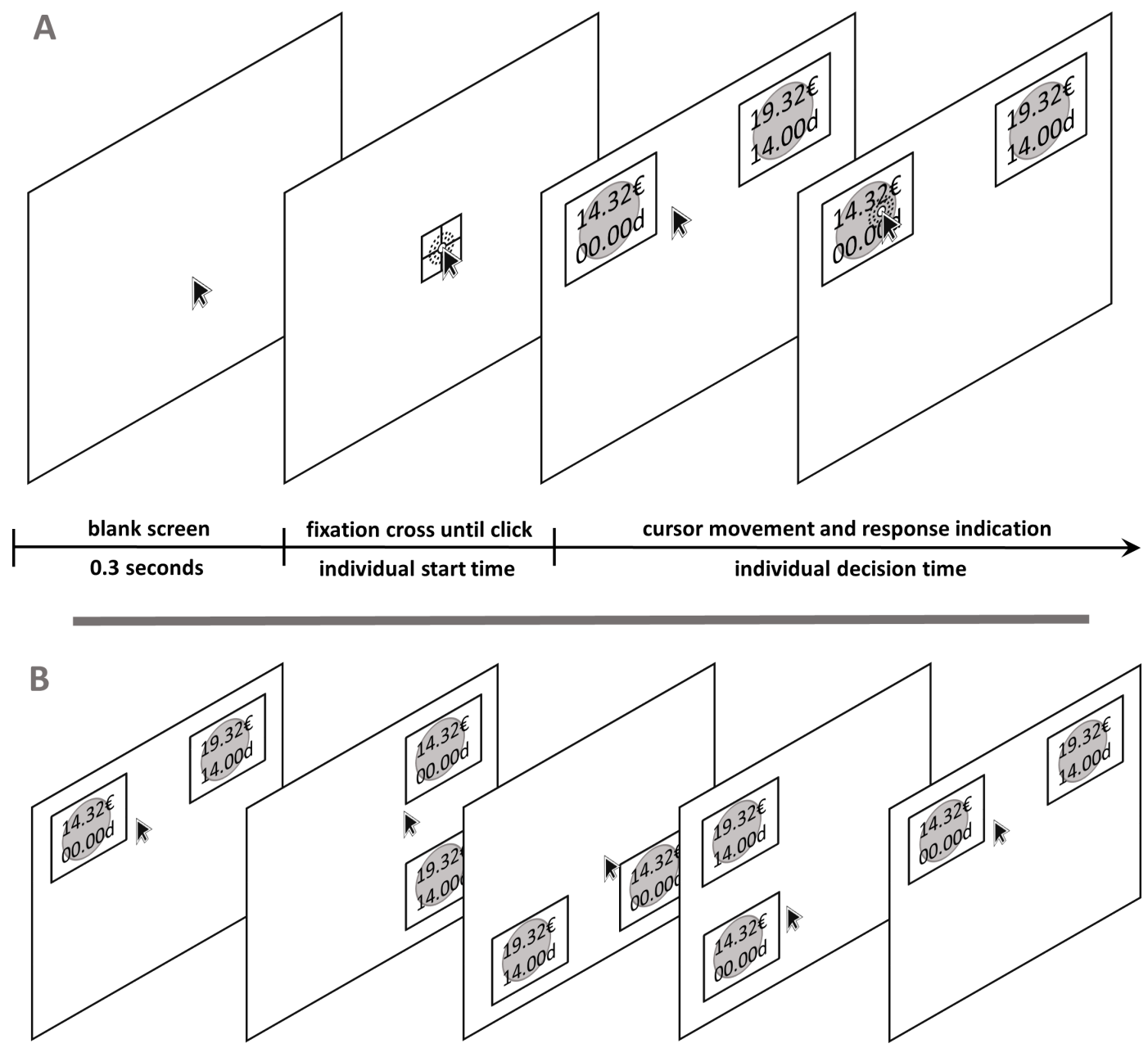

Figure 2: Trial protocol of Experiment 1. A: Each trial started with a blank screen ( 0.3 seconds), followed by the presentation of the fixation cross, which had to be clicked for a self-paced start of the mouse cursor tracking, and hence, the presentation of the choice options. After the presentation of the choice options, participant indicated their choice by clicking in the circular, grey areas underlying each option. B: Clock-wise variation of the position of the choice options, and hence, response areas.

\subsubsection{Design}

In ITC, the relationship between the two options can be described in terms of intervals and ratios. The reward ratio is given as the ratio of the rewards of both options (i.e., reward of the SS option divided by the reward of the LL option), whereas the interval is given by the difference of the delays of both options (i.e., delay of the SS option subtracted from the delay of the LL option). We used such intervals and reward ratios to construct the design of the study. 
The paradigm was split into two different blocks: a measurement block intended to measure participants' individual temporal discounting, and an experimental block intended to capture choice repetition. The measurement block consisted of one set of trials (taken from Dshemuchadse et al., 2013) with a wide range of combinations by varying the intervals $(1,2,3,5,7,10$, and 14 days), reward ratios $(20,50,70,80,88,93,97$, and 99\%), the delay of the sooner option ( 0 and 7 days), and the reward of the late option ( $€ 19.68$ and $€ 20.32$ ). The order of the resulting 224 trials was randomized across participants.

We used the choice data of this measurement block to estimate participants' individual temporal discounting in the form of indifference points. Indifference points describe the specific reward ratio where both options are equally attractive to the participant, that is, the probability of choosing either option is 50\%. For each participant, we calculated indifference points for each interval by fitting a logistic function to the choice data and taking the point of inflection (Scherbaum et al., 2013). Based on these estimated indifference points, we interpolated the indifference points for the remaining intervals ${ }^{3}$. As a result, we had estimates of indifference points for each interval between 1 and 14 days, which we then used to construct trials in the subsequent experimental block.

The experimental block consisted of a set of individually tailored trials with a sequential manipulation to capture choice history biases. In each sequence, twelve trials were presented in which a strong preference toward the SS option is gradually changed into strong preference towards the LL option, or vice versa. The preference in a trial can be described as the difference between the reward ratio and the indifference point for the specific interval, which we denote as distance. Thus, a negative distance represents a preference for the LL option, a positive distance represents a preference for the SS option, and a zero distance matches the indifference point which represents by definition no preference toward either option.

For each sequence of trials, we randomly chose the reward of the LL option ( $€ 19.68$ or $€ 20.32)$ and the delay of the SS option ( 0 or 7 days). For each trial within the sequence, the delay of the LL option was randomly chosen from the set of intervals at which the respective distance and indifference point produced a valid reward ratio (i.e., $0 \leq$ reward ratio $\leq 1)$. The reward of the SS option was then calculated. Furthermore, we varied the direction of these sequences (direction $=$ ascending vs descending) and created eight sequences for each direction consisting of 12 distances $(-0.30,-0.25,-0.19,-0.14,-0.08,-0.03,0.03,0.08,0.14,0.19,0.24,0.30)$. Hence, the experimental block consisted of 192 trials. The order of the 16 sequences was randomized across participants.

In order to obtain a sufficient level of randomness in those individually tailored sequences, we only included participants showing temporal discounting that comprised at least one valid distance manipulation in each direction across intervals (see Procedure).

In sum, participants completed 416 trials during the experiment. The first 224 trials were meant to measure participants' individual temporal discounting. The last 192 trials consisted of 16 individually tailored trial sequences in which participants' preference was either gradually manipulated from the SS option towards the LL option (descending, 8 sequences $=96$ trials), or vice versa, that is, from the LL option toward the SS option (ascending, 8 sequences $=96$ trials).

\subsubsection{Preprocessing and statistical analyses}

To examine participants' temporal discounting in the measurement block, we extracted individual $k$ parameters (Green, Fristoe, et al., 1994) from their choices by fitting a hyperbolic function to each participant's indifference points over intervals ${ }^{4}$. As an estimate of the indifference points, we determined the point of inflection of a logistic function that was fitted $^{5}$ the individual choices as a function of increasing reward ratios. We chose the hyperbolic function and the $k$ parameter to preserve comparability with our previous work (viz. Dshemuchadse et al., 2013) and because it offers a parsimonious summary of discounting in a single parameter. Hence, it serves our aim to characterize decision behavior quantitatively rather than comparing different models (for a range of different models and model comparison, see Doyle, 2013; Wulff \& van den Bos, 2017). However, to enhance comparability within and between studies, we also calculated

\footnotetext{
${ }^{3}$ We interpolated in the following ways: If the measured indifference points were monotonically decreasing, we interpolated cubically using the 'pchip' method within the function 'interp1' in Matlab. If the measured indifference points were not monotonically decreasing, we fitted them to an exponential and a hyperbolic model. The model that provided the better fit (as quantified by standard errors) was used to interpolate the indifference points.

${ }^{4}$ The fitting of the hyperbolic function was performed by applying Matlab's multidimensional unconstrained nonlinear minimization function to the hyperbolic function $1 /(1+k * x)=y$, with $x$ denoting time interval, $y$ denoting subjective value, and $k$ denoting the discounting parameter.

${ }^{5}$ The fitting of the logistic regression model was performed using the function 'logitfit' from the StixBox mathematical toolbox by Anders Holtsberg (www.maths.lth.se/matstat/stixbox/). The fit was based on the model $\log [p /(1-p)]=b_{0}+x \times b_{1}$, where $p$ is the probability that the choice is 1 (SS option) and not 0 (LL option), $x$ represents value differences, and $b_{0}$ and $b_{1}$ represent the point estimates for the logistic function.
} 
the area under the curve (AUC) from the proportion of LL choices for each interval as a model-free measure of temporal discounting that bypasses several caveats of the $k$ parameter with respect to distribution and sensitivity (Myerson et al., 2001).

\subsection{Results}

\subsubsection{Manipulation check}

In the measurement block, participants completed 224 trials. The aim of the measurement block was twofold: first, with respect to the experimental design, to measure participants' individual temporal discounting in order to individually tailor trial sequences for the experimental block (see Design); second, to run a general manipulation check of our version of a temporal discounting task indicated by participants' choices and choice dynamics ( $\mathrm{H} 1$ and $\mathrm{H} 2$ ). Hence, we will use the trials from the measurement block only for the subsequent analyses. To evaluate participants' temporal discounting (H1), we examined their individual $k$ parameters. We found average $k$ parameters of the hyperbolic discounting curve of $0.17(S D=0.17,95 \%$ bCI $=[0.12,0.24]$, see Figure $3 \mathrm{~A})$. Overall, the hyperbolic model produced a good model fit indicated by a high average $R^{2}$ of $.88(S D=.16,95 \% \mathrm{bCI}=[.79, .92]) .{ }^{6}$ For the model-free measure of temporal discounting, we found average AUC of $.30(S D=.14,95 \% \mathrm{bCI}=[.27, .35])$. Hence, we found similar discounting as in Dshemuchadse et al. (2013), confirming Hypothesis 1.
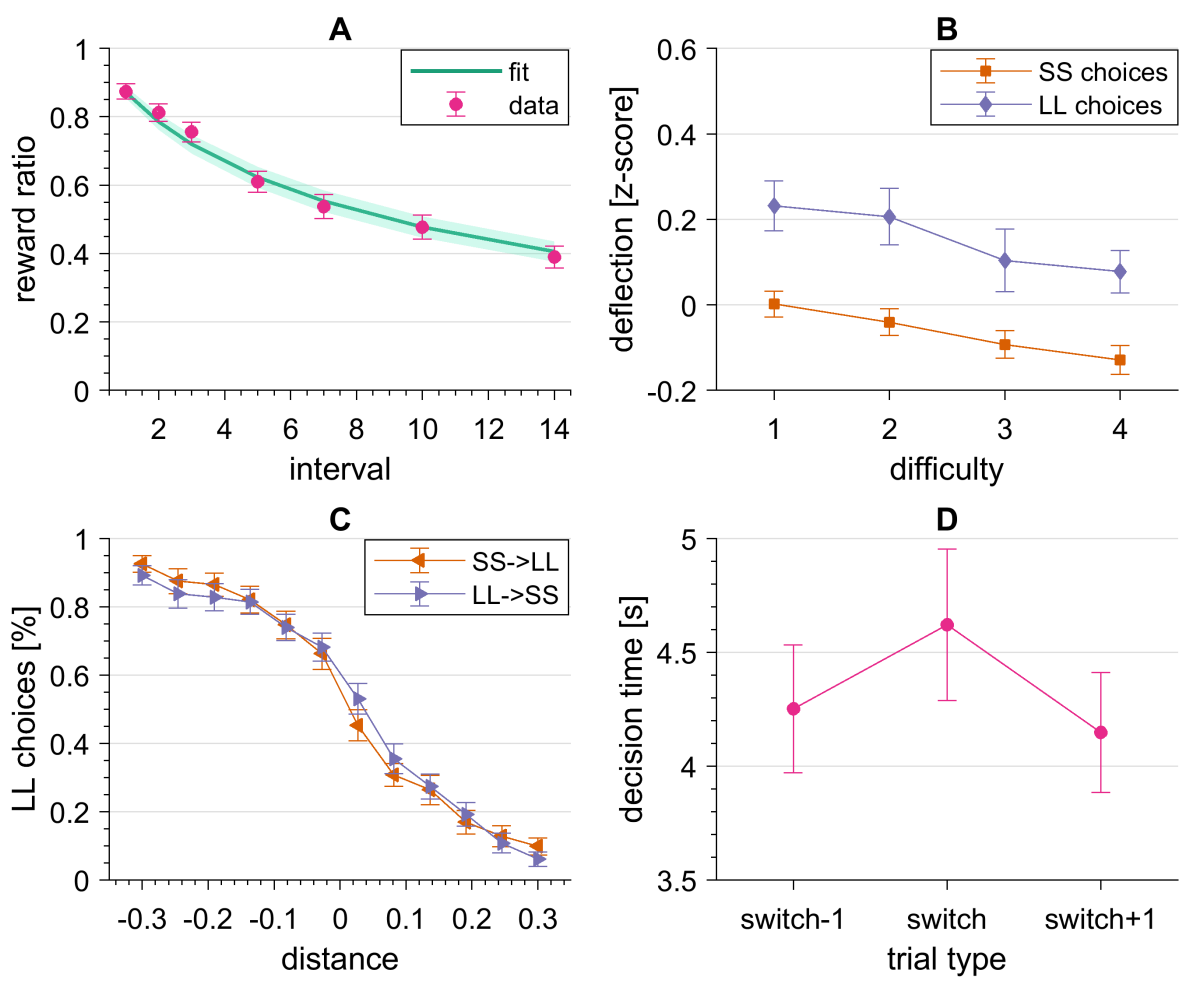

Figure 3: Results overview of Experiment 1. A: Mean indifference points depicting the decrease in reward ratio as a function of interval. The curve displays the fitted hyperbolic function. B: Mean deflections of the cursor trajectories as a function of difficulty, separately for SS and LL choices. C: Mean proportion of LL choices as a function of distance, separately for SS and LL choices. D: Mean decision times as a function of trial type (pre-switch, switch, post-switch). Note: Error bars and error areas depict standard errors in all plots.

To evaluate participants' choice dynamics (H2), we investigated the influence of the decision difficulty on deflection in the cursor trajectories. We operationalized decision difficulty as the distance of the trial's reward ratio from the respective indifference point at the trial's interval. Hence, for the measurement block, decision difficulty denotes

\footnotetext{
${ }^{6}$ The fit of the hyperbolic function was based on minimizing the summed squared errors $(S S E) . R^{2}$ is defined as the ratio of the sum of squares of the regression ( $S S R)$ and the total sum of squares $(S S T)$. Since $S S T$ is defined as $S S R+S S E, R^{2}$ is defined by $1-S S E / S S T$.
} 
the same as distance for the experimental block, except that decision difficulty was determined post-hoc and then separated into quartiles. A repeated-measures ANOVA on deflection with the independent variables difficulty and choice (SS vs LL) revealed significant main effects of difficulty, $F(3,102)=4.98, p=.003, \eta^{2}=.12$, and choice, $F(1,34)=14.47, p<.001, \eta^{2}=.30$, but no interaction, $F(3,102)=0.24, p=.87$ (Figure 3B). Hence, except for the interaction, we found similar choice dynamics as in the Dshemuchadse et al. (2013), largely confirming Hypothesis 2.

\subsubsection{Choice history bias}

In the experimental block, participants completed 192 trials consisting of eight 12 -step sequences in each direction. The aim of the experimental block was to manipulate participant's preference for the SS option toward preference for the LL option, and vice versa, in order to investigate choice repetition. Hence, we will use the trials from the experimental block only for the subsequent analyses.

To investigate choice history bias (H3), we investigated the influence of the distance and the direction on the choice ratios. A repeated-measures ANOVA on the ratio of LL choices with the independent variables distance and direction revealed a significant main effect of distance, $F(11,396)=157.69, p<.001, \eta^{2}=.81$, but neither a main effect of direction, $F(1,36)=0, p=1.0$, nor an interaction, $F(11,39)=1.74, p=.06$ (Figure 3C). The results of the ANOVA were corroborated by a Generalized Linear Mixed Model (GLMM) analysis; please find all details and results in the Appendix. We also summarized choice ratios into a choice history bias index by calculating the difference between participants' average ratio of LL choices of either direction. A positive bias index would indicate choice repetition which we could not find applying an one-sided $t$-test, $t(36)=0, p=.5$. Hence, we did not find choice history bias as expected, rejecting our Hypothesis 3.

As the analysis of the choice data did not show the results we had expected, we checked whether participants were at least sensitive to our sequential manipulation of their preference. If so, we should find longer decision times in trials in which participants switched from choosing the initially preferred option to the alternative option compared to pre-switch and post-switch trials (H4). A one-factorial repeated-measures ANOVA on decision times revealed a significant main effect of trial type, $F(2,72)=10.52, p<.001, \eta^{2}=.23$ (Figure 3D). Post-hoc tests (Holm corrected) revealed significant differences in decision times between pre-switch and switch trials, $p_{\text {holm }}=.006, d=-0.53$, as well as switch and post-switch trials, $p_{\text {holm }}<.001, d=0.65$. Hence, we found decision times that developed over the course of the trial sequence as expected, confirming Hypothesis 4.

\subsection{Discussion}

From the results of Experiment 1, we learned that our adapted version of the mouse-based ITC paradigm produced effects ( $\mathrm{H} 1$ and $\mathrm{H} 2)$ related to temporal discounting that are similar to findings from previous studies using a similar procedure and the same range of rewards and delays (Dshemuchadse et al., 2013; Scherbaum et al., 2012). However, our paradigm did not reliably elicit choice repetition (H3) similar to our previous studies (e.g., Scherbaum et al., 2016), though the analysis of decision times (H4) indicated that participants were at least sensitive to our trial sequences (Senftleben et al., 2020; Senftleben et al., 2019).

Based on this inconclusive pattern of results, we asked for the reasons that the paradigm showed every effect as expected except for the choice history bias. A sensitivity analysis excluded the power of the study as a potential cause (see Participants), and thus, we reviewed commonalities and differences between the paradigm at hand and the gamified delay discounting task that had reliably elicited choice history biases (Scherbaum et al., 2016; Schoemann \& Scherbaum, 2020; Senftleben et al., 2020; Senftleben et al., 2019). We identified two features of the gamified task that were not implemented in our current ITC paradigm, namely, a fully controlled, short inter-trial interval and time pressure. Since we had previously found that the length of the ITI is an effective modulator of the choice history bias (Senftleben et al., 2020; Senftleben et al., 2019), we decided to adapt the paradigm to control this modulator.

\section{Experiment 2}

In our second experiment, we ran an adapted version of the mouse-based ITC task from the first experiment with the same aims, hypotheses (i.e., Hypotheses 1-4 in Experiment 1), and stimuli (i.e., range of rewards and delays). As concluded from Experiment 1, we implemented a controllable and short inter-trail interval as well as time pressure. For the controllable inter-trial interval, we substituted the complete self-paced trial start with a variant in which participants could start the trial by themselves within a specified time limit (otherwise the trial started automatically after this time limit had expired). This variant did not give us full control over the inter-trial interval, but set an upper limit. For the 
time pressure, we instructed participants to complete their choices in trials within a specified deadline and visualized this deadline during trials; if the deadline had expired, the trial was aborted.

\subsection{Method}

\subsubsection{Participants}

We recruited fifty participants ( $66 \%$ female, mean age $=26.56$ years, range $=20-49)$ through the department's data-base system ORSEE (Greiner, 2015). The experiment was conducted at the Technische Universität Dresden and lasted approximately 45 minutes; participants gave informed consent and received either class credits or $€ 5.00$ show-up fee; all participants had normal or corrected-to-normal vision. According to experimental protocol, similar to Experiment 1, fifteen participants had to finish the experiment after the first block due to insufficient temporal discounting (see the Procedure section in Experiment 1). Hence, we achieved a final sample size of thirty-five participants (65.71\% female, mean age $=26.71$ years, range $=20-49$ ) for all subsequent analyses (for a justification of the sample size, please see the Participants section in Experiment 1).

\subsubsection{Apparatus, stimuli, and design}

The setup followed the setup of Experiment 1. Neither apparatus, nor stimuli, nor design were changed. However, in order to visualize newly implemented time limits (see Procedure), we additionally presented colored (i.e., green and red), decreasing and increasing squares (see Figure 4).

\subsubsection{Procedure}

Again, participants were asked to decide on each trial which of two options (SS vs LL) they preferred. The experimental procedure was essentially unchanged, with the exception that we implemented time limits in both stages. In the start stage, participants were instructed to click on the fixation cross within a time limit of 2 seconds, ensuring a maximum inter-trial interval of 2.3 seconds (see Figure 4). During the second stage, participants had to indicate their choice by clicking on either response area within a time limit of 4 seconds. If participants missed the time limit in the start stage, the trial started automatically as if participants had clicked on the fixation cross. If participants missed the time limit in the response stage, the next trial started with the presentation of the fixation cross.

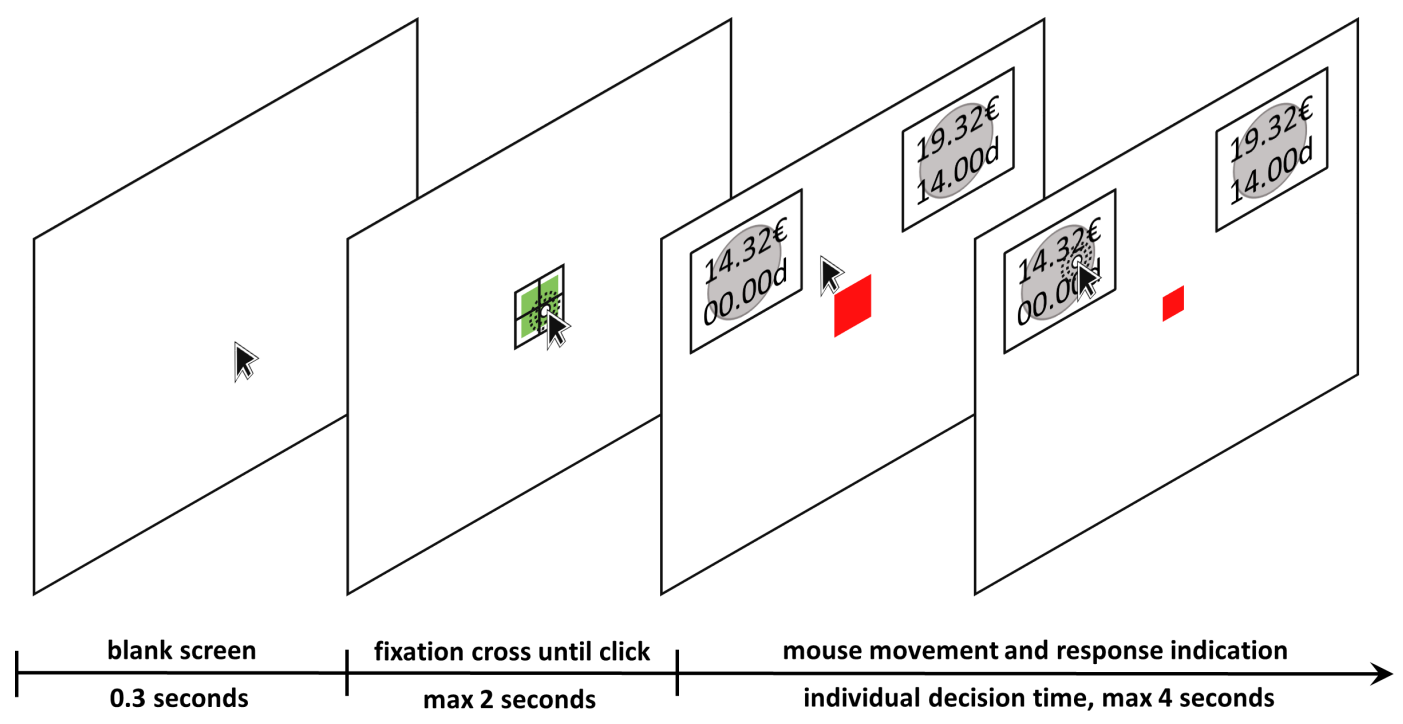

Figure 4: Trial protocol of Experiment 2. Each trial started with a blank screen ( 0.3 seconds), followed by the presentation of the fixation cross underlayed with a filled green square, which had to be clicked for a self-paced start of the mouse cursor tracking within 2 seconds, and hence, the presentation of the choice options. The green square diminished over time visualizing the time limit for the start stage. After the presentation of the choice options, participant indicated their choice by clicking in the circular, grey areas underlying each option. The presentation of the choice options was accompanied by the presentation of diminishing red square in the center of the screen visualizing the time limit of the response stage. 


\subsubsection{Preprocessing and statistical analyses}

We evaluated the data of Experiment 2 with exactly the same analyses as in Experiment 1. Thus, we do not provide further explanations; please consult the Results section of Experiment 1 for clarifications.

\subsection{Results}

\subsubsection{Manipulation check}

With regard to temporal discounting (H1), the choice data revealed an average $k$ parameters of the hyperbolic discounting curve of $0.23(S D=0.18,95 \% \mathrm{bCI}=[0.18,0.30]$, see Figure 5A). Overall, the hyperbolic model produced a good model fit indicated by a high average $R^{2}$ of $.82(S D=.17,95 \% \mathrm{bCI}=[.75, .86])$. For the model-free measure of temporal discounting, we found average AUC of $.27(S D=0.11,95 \% \mathrm{bCI}=[0.23,0.30])$. Hence, we found similar discounting as in Dshemuchadse et al. (2013) confirming our Hypothesis 1, and replicating temporal discounting from Experiment 1.
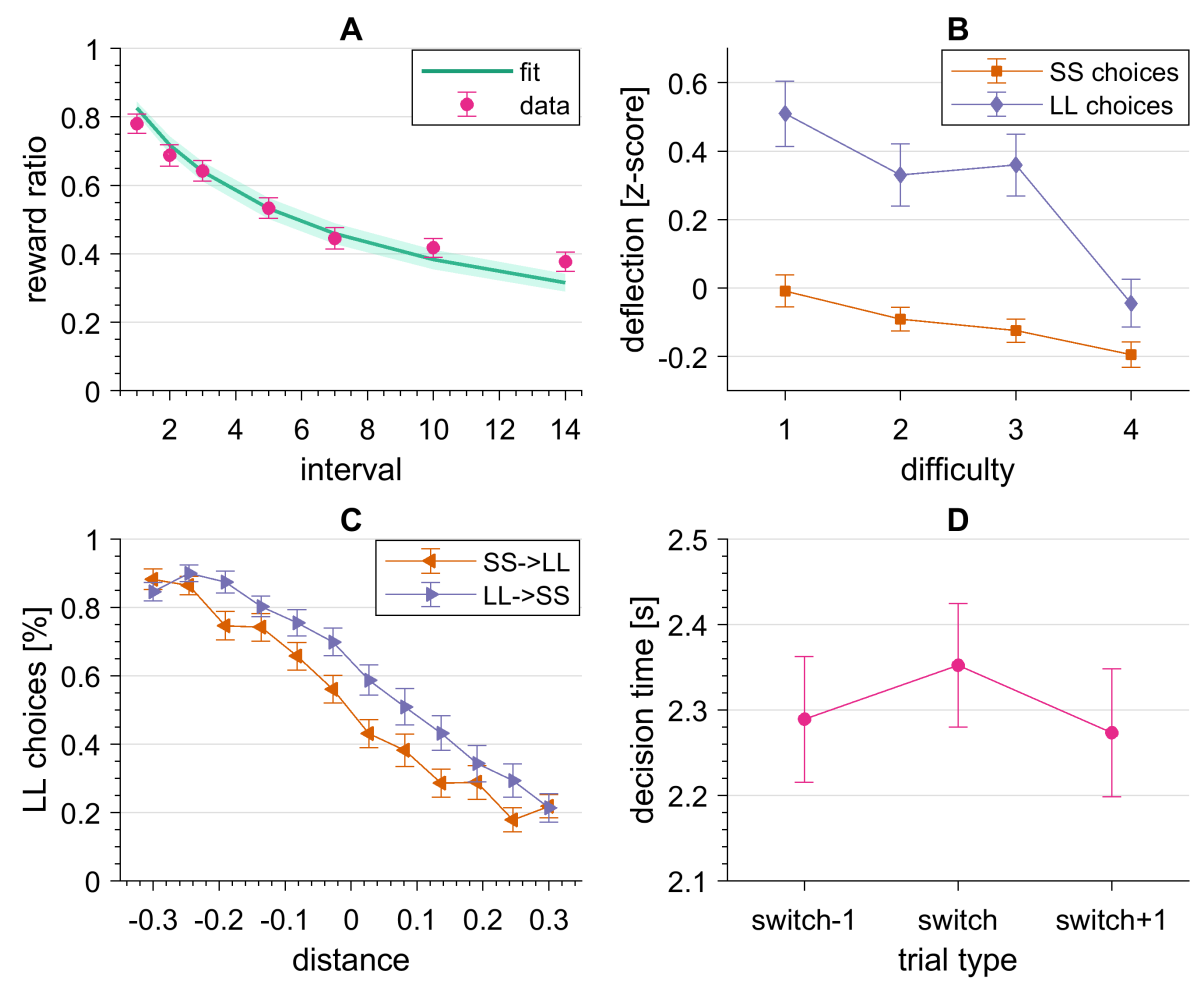

Figure 5: Results overview of Experiment 2. A: Mean indifference points depicting the decrease in reward ratio as a function of interval. The curve displays the fitted hyperbolic function. B: Mean deflections of the cursor trajectories as a function of difficulty, separately for SS and LL choices. C: Mean proportion of LL choices as a function of distance, separately for SS and LL choices. D: Mean decision times as a function of trial type (pre-switch, switch, post-switch). Note: Error bars and error areas depict standard errors in all plots.

With regard to mouse cursor trajectories (H2), a repeated-measures ANOVA on deflection with the independent variables difficulty and choice revealed significant main effects of difficulty, $F(3,93)=12.22, p<.001, \eta^{2}=.28$, and choice, $F(1,31)=14.32, p<.001, \eta^{2}=.31$, as well as a significant interaction, $F(3,93)=3.49, p=.02, \eta^{2}=.10$, see Figure 5B. Hence, we found similar choice dynamics as in the Dshemuchadse study (2013), confirming our Hypothesis 2 and replicating the main effects from Experiment 1.

\subsubsection{Choice history bias}

With regard to choice history bias (H3), a repeated-measures ANOVA on the ratio of LL choices with the independent variables distance and direction revealed significant main effects of distance, $F(11,374)=114.19, p<.001, \eta^{2}=.77$, 
and direction, $F(1,34)=17.48, p<.001, \eta^{2}=.34$, as well as a significant interaction, $F(11,374)=3.48, p<$ $.001, \eta^{2}=.09$, see Figure 5C. Again, the results of the ANOVA were corroborated by a Generalized Linear Mixed Model (GLMM) analysis; please find all details and results in the Appendix. We also found a significant bias index, $t(34)=4.18, p<.001, d=0.74,95 \% \mathrm{bCI}=[0.44,1.04]$. Hence, we found a choice history bias as expected, confirming our Hypothesis 3.

With regard to decision conflict (H4), a one-factorial repeated-measures ANOVA on decision times revealed a significant main effect of trial type, $F(2,72)=16.57, p<.001, \eta^{2}=.33$, see Figure 5D. Post-hoc tests (Holm corrected) revealed significant differences in decision times between pre-switch and switch trials, $p_{\text {holm }}<.001, d=-0.76$, as well as switch and post-switch trials, $p_{\text {holm }}<.001, d=0.87$. Hence, we found decision times that developed over the course of the trial sequence as expected, confirming our Hypothesis 4 and replicating the effect from Experiment 1.

\subsection{Discussion}

The results in Experiment 2 showed exactly the pattern that we expected and that is well in line with our previous research. We indeed showed that the choice history bias which we repeatedly showed in our gamified delay discounting task also generalizes to a classic intertemporal choice paradigm. Taking Experiment 1 and 2 together, the findings additionally support another effect that we observed previously: The choice history bias is influence by the inter-trial interval (Senftleben et al., 2020; Senftleben et al., 2019). To test whether the introduced shorter inter-trial interval we introduced in Experiment 2 was indeed responsible for eliciting the choice history bias and to replicate the choice history bias in our novel ITC paradigm, we planned a third, preregistered experiment.

\section{Experiment 3}

As Experiment 1 and 2 were more of an exploratory nature showing the choice history bias in ITC for the first time and suggesting a modulation by the inter-trial interval (ITI), we headed for a preregistered validation study. In such a series of consecutive experiments, we certainly had the same four hypotheses as for Experiments 1 and 2. However, for the sake of simplifying the preregistration of Experiment 3 (see osf.io/57qpm/), we chose only to include our novel hypotheses that we derived from the results obtained in Experiment 1 and 2. First, we expected a bias index greater than zero for choice behavior in the short ITI condition (PH1). Second, we expected a higher bias index in the short ITI condition than in the long ITI condition (PH2).

\subsection{Method}

\subsubsection{Participants}

We based our sample size rational on a priori power analysis with $\mathrm{G}^{*}$ Power (Faul et al., 2009). We needed a sample size of $N=45$ to detect a medium sized effect of $d=0.5$ with high power of $1-\beta=.95$. We knew from Experiment 1 and 2 that some participants will show insufficient temporal discounting in the measurement block, and would hence not, complete the experimental block. Therefore, we planned to keep on recruiting participants until we had 45 complete data sets.

We recruited fifty-four participants $(70.37 \%$ female, mean age $=24.41$ years, range $=18-67)$ through the department's data-base system ORSEE (Greiner, 2015). The experiment was conducted at the Technische Universität Dresden and lasted approximately 60 minutes; participants gave informed consent and received either class credits or $€ 6.00$ show-up fee; all participants had normal or corrected-to-normal vision. According to the preregistered experimental protocol, similar to Experiment 1 and 2, nine participants had to finish the experiment after the first block due to insufficient temporal discounting (see Procedure section in Experiment 1). Unexpectedly, and only revealed during data analysis, one participant chose the LL option throughout the complete experimental block and was discarded. Hence, we achieved a final sample size of forty-four participants $(68.18 \%$ female, mean age $=24.84$ years, range $=18-67)$ for all subsequent analyses, which constituted a slight deviation from our preregistered sampling plan, and hence a slightly reduced expected power $(1-\beta=.947)$ as revealed by a post-hoc sensitivity analysis.

\subsubsection{Apparatus and stimuli}

The setup followed the setup of Experiment 1 and 2. Neither apparatus, nor stimuli were changed. Again, we presented decreasing and increasing squares in order to visualize time limits (see Procedure). 


\subsubsection{Procedure}

Again, participants were asked to decide on each trial which of two options (SS vs LL) they preferred. The experimental procedure was essentially unchanged, with the exception that we implemented a new procedure of the start stage. One version of the start stage procedure was implemented exactly the same way as in Experiment 2 in such a way that participants were instructed to click on the fixation cross within a specified time limit ( 1 second, see Figure 6 and Design). In the new version of the start stage procedure, participants were instructed to click on the fixation cross after a specified time limit (3 seconds). This new procedure served to implement a long ITI.

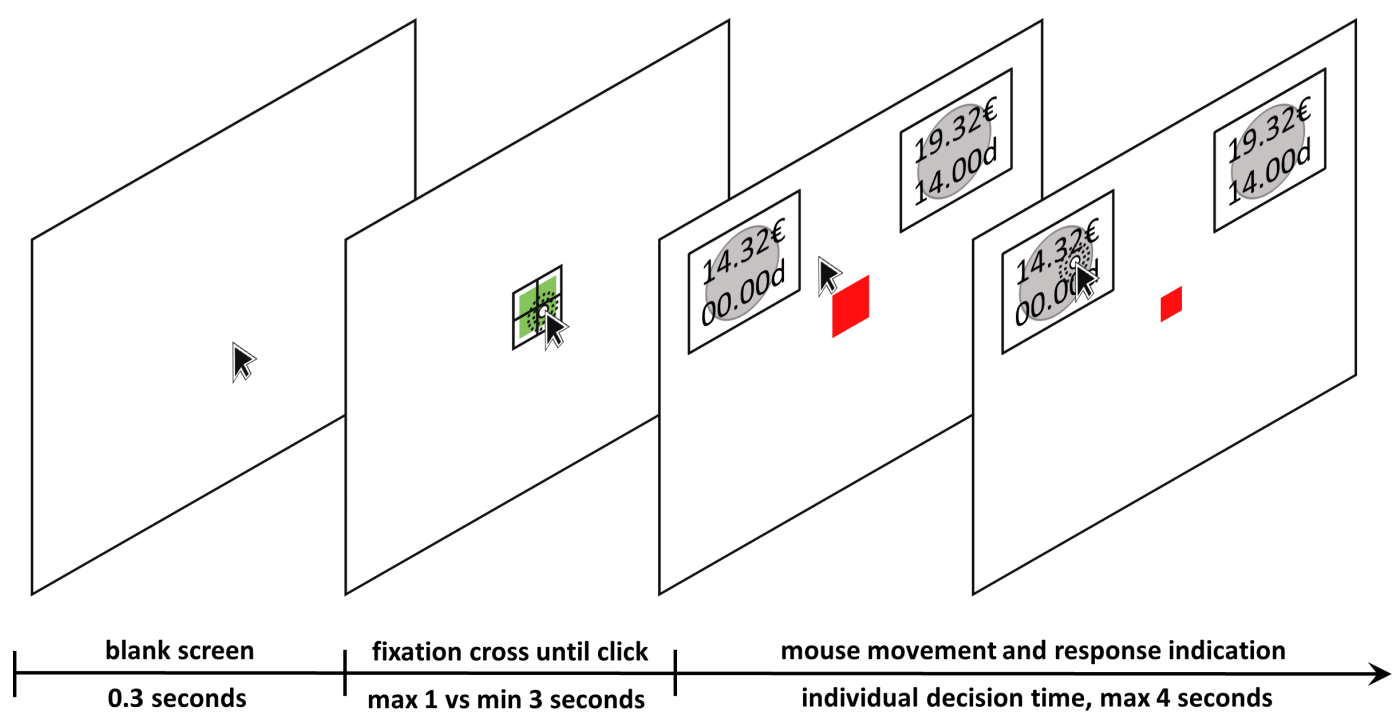

Figure 6: . Trial protocol of Experiment 3. Each trial started with a blank screen ( 0.3 seconds), followed by the presentation of the fixation cross. In the short ITI condition, the fixation cross was underlayed with either a filled green square that diminished over time and had to be clicked for a self-paced start of the mouse cursor tracking within 1 second, and hence, the presentation of the choice options. In the long ITI condition, the fixation cross was underlayed with either an empty white square that filled over time green and had to be clicked for a self-paced start of the mouse cursor tracking after 3 seconds, and hence, the presentation of the choice options. After the presentation of the choice options, participant indicated their choice by clicking in the circular, grey areas underlying each option. The presentation of the choice options was accompanied by the presentation of diminishing red square in the center of the screen visualizing the time limit of the response stage.

\subsubsection{Design}

The general design of Experiment 3 was similar to the design of Experiment 1 and 2 with the exception that we introduced a second experimental block and shortened the length of the individually tailored trial sequences. Hence, Experiment 3 consisted of three blocks: One measurement block and two experimental blocks. The measurement block was designed exactly the same way as in Experiment 1 and 2. In the slightly shorter version of the experimental blocks, we used ten distances $(-0.25,-0.19,-0.14,-0.08,-0.03,0.03,0.08,0.14,0.19,0.25)$ instead of 12 distances (see Design section in Experiment 1) to sequentially manipulate participants' preference toward the options.

In sum, participants completed 608 trials during the experiment. The first 224 trials were meant to measure participants' individual temporal discounting. The last 320 trials were equally distributed between the two (shorter) experimental blocks consisting of 16 individually tailored trial sequences in which participants' preference was either manipulated from the SS option toward the LL option (descending, 8 sequences $=80$ trials), or vice versa, that is, from the LL option toward the SS option (ascending, 8 sequences $=80$ trials), respectively.

The only systematic variation between the two experimental blocks consisted of the manipulation of the ITI (short vs long). The sequential order of the experimental blocks was counterbalanced between participants. Please note that the measurement block (short ITI with 2 seconds time limit) poses a direct replication of the same block in Experiment 2. 


\subsection{Results}

We largely evaluated the data of Experiment 3 with the same analyses as for Experiment 1 and 2. Therefore, we do not provide further explanations, except were necessary due to new manipulations; please consult the Results section of Experiment 1 for clarifications.

Due to the constraints when reporting the results of a preregistered study, we report the confirmatory analysis, first, and turn to the exploratory analysis, second. Please note that a large part of the exploratory analyses tests Hypothesis 1-4 that were also examined in Experiment 1 and 2, and hence, the exploratory part also contains confirmatory analyses, even though convention asks to call it exploratory.

\subsubsection{Confirmatory analyses}

To test our preregistered hypotheses (PH1 and $\mathrm{PH} 2)$, we calculated the bias index for the two experiment blocks separately. We found a significant bias index, $t(43)=1.72, p=.04, d=0.26,95 \% \mathrm{bCI}=[-0.03,0.58]$, in the short ITI condition, confirming PH1. In contrast, we found a non-significant bias index, $t(43)=1.32, p=.10$, in the long ITI condition. Comparing the two ITI conditions with regard to choice history bias, we found that the bias index in the short ITI condition was not greater than the bias index in the long ITI condition, $t(43)=0.37, p=.36$, contradicting $\mathrm{PH} 2$.

\subsubsection{Exploratory analyses}

Manipulation check With regard to temporal discounting (H1), the choice data revealed an average $k$ parameters of the hyperbolic discounting curve of $0.28(S D=0.34,95 \% \mathrm{bCI}=[0.21,0.43]$, see Figure $7 \mathrm{~A})$. Overall, the hyperbolic model produced a good model fit indicated by a high average $R^{2}$ of $.90(S D=.10,95 \% \mathrm{bCI}=[.86, .92])$. For the model-free measure of temporal discounting, we found average AUC of .25 ( $S D=.13,95 \% \mathrm{bCI}=[0.21,0.29])$. Hence, we found similar discounting as in Dshemuchadse et al. (2013) confirming our Hypothesis 1 and replicating temporal discounting from Experiment 1 and 2.

With regard to mouse cursor trajectories (H2), a repeated-measures ANOVA on deflection with the independent variables difficulty and choice revealed significant main effects of difficulty, $F(3,108)=9.71, p<.001, \eta^{2}=.21$, and choice, $F(1,36)=24.30, p<.001, \eta^{2}=.40$, but no interaction, $F(3,108)=1.19, p=.32$, see Figure 7B. Hence, we found similar choice dynamics as in Dshemuchadse et al. (2013), confirming our Hypothesis 2 and replicating the effects pattern from Experiment 1 and the main effects from Experiment 2.

In this experiment, we aimed to manipulate the ITI, which we implemented through different start procedures (see Procedure). Hence, we additionally tested whether the different start procedures indeed cause different ITIs. Participants had an average ITI of 0.59 seconds $(S D=0.19)$ in in the short ITI condition, and an average ITI of 3.38 seconds $(S D=0.13)$ in the long ITI condition, which indeed differed significantly, $t(43)=-101.67, p<.001, g=$ $-16.88,95 \%$ bCI $=[-21.20,-14.47]$.

Choice history bias With regard to choice history bias (H3), an additional repeated-measures ANOVA on the ratio of LL choices with the independent variables ITI, distance, and direction revealed significant main effects of distance, $F(9,387)=177.66, p<.001, \eta^{2}=.81$, as well as a significant interaction direction $\times$ distance, $F(9,387)=2.13, p=.03, \eta^{2}=.05$. All other model terms were non-significant, including the theoretically important interaction ITI $\times$ direction $\times$ distance interaction, $F(9,387)=1.62, p=.107, \eta^{2}=.04$, , see Figure $7 \mathrm{C}$ and E. As in Experiment 1 and 2, the results of the ANOVA were corroborated by a Generalized Linear Mixed Model (GLMM) analysis; please find all details and results in the Appendix. Hence, this additional analysis supports the results from the confirmatory analysis as well as confirms Hypothesis 3 in such a way that we found choice repetition for the short ITI condition only and that the ITI manipulation did not elicit quantitatively different choice repetition.

With regard to decision conflict $(\mathrm{H} 4)$, a two-factorial repeated-measures ANOVA (ITI $\times$ trial type) on decision times revealed significant main effects of ITI, $F(1,43)=16.59, p<.001, \eta^{2}=.28$, and trial type, $F(2,86)=18.54, p<$ $.001, \eta^{2}=.30$, but no significant interaction, $F(2,86)=2.65, p=.077$, , see Figure 7D and F. For the short ITI condition, post-hoc tests (Holm corrected) revealed significant differences in decision times between pre-switch and switch trials, $p_{\text {holm }}=.032, d=-0.38$, switch and post-switch trials, $p_{\text {holm }}<.001, d=0.67$, as well as pre-switch and post-switch trials, $p_{\text {holm }}=.032, d=0.38$. For the long ITI condition, post-hoc tests (Holm corrected) revealed significant differences in decision times between switch and post-switch trials, $p_{\text {holm }}<.001, d=0.65$, as well as pre-switch and post-switch trials, $p_{\text {holm }}=.003, d=0.53$, but no difference between pre-switch and switch trials, $p_{\text {holm }}=.884, d=-0.38$. Hence, we found decision times that developed over the course of the trial sequence as expected, confirming our Hypothesis 4 and replicating the effect from Experiment 1 and 2. 
A

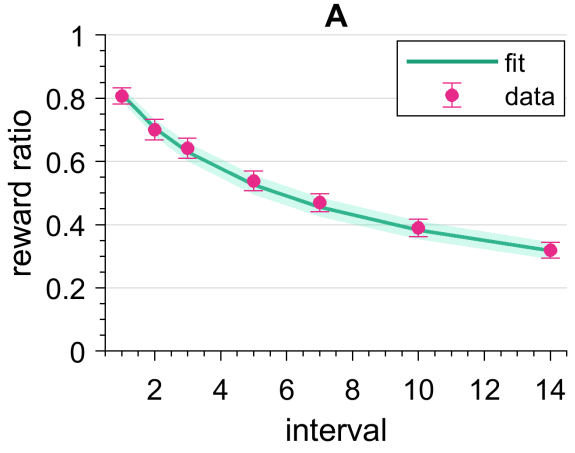

C

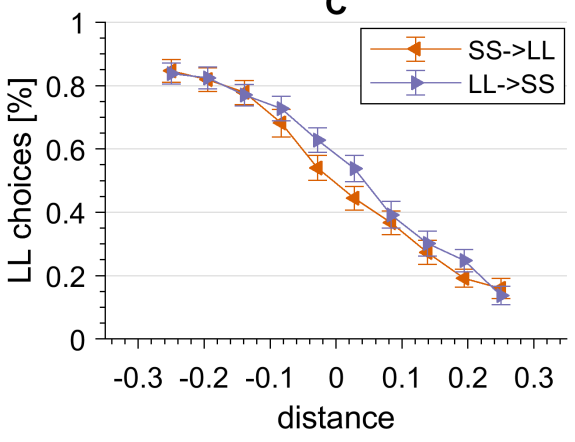

E

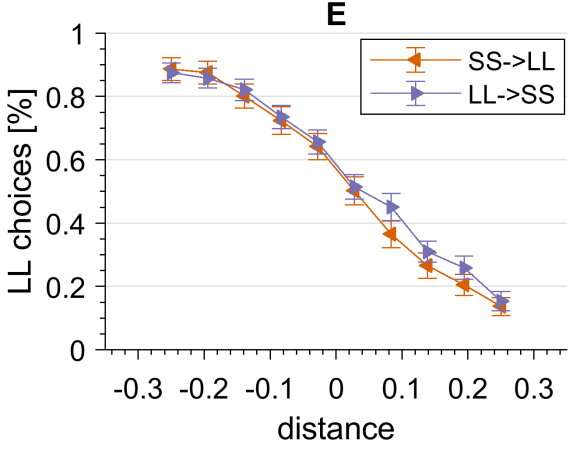

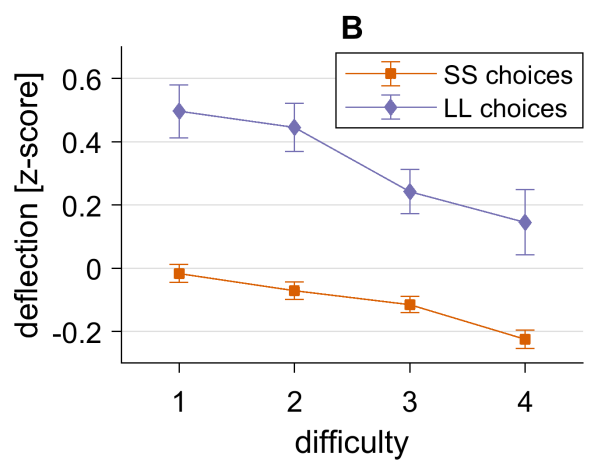

D
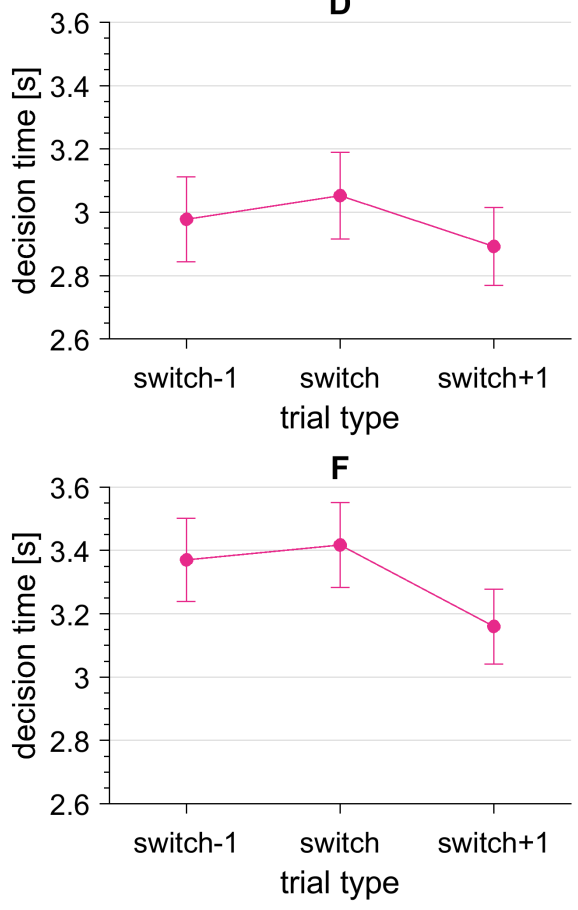

Figure 7: Results overview of Experiment 3. A: Mean indifference points depicting the decrease in reward ratio as a function of intervals. The curve displays the fitted hyperbolic function. B: Mean deflections of the cursor trajectories as a function of difficulty, separately for SS and LL choices. C: Mean proportion of LL choices as a function of distance, separately for SS and LL choices, for the short ITI condition. D: Mean decision times as a function of trial type (pre-switch, switch, post-switch), for the short ITI condition. E: Mean proportion of LL choices as a function of distance, separately for SS and LL choices, for the long ITI condition. F: Mean decision times as a function of trial type (pre-switch, switch, post-switch), for the long ITI condition. Note: Error bars and error areas depict standard errors in all plots. 


\subsection{Discussion}

The results in Experiment 3 showed the pattern that we expected to a certain degree only. We found a choice history bias in series of choices with a short ITI (PH1), and no choice history bias in series of choices with a long ITI. However, these effects merely differed qualitatively rather than quantitatively (PH2). In addition to those findings, Experiment 3 replicated Experiment 1 and 2 with regard to choice (dynamics) in intertemporal decision making which speaks in favor of the overall validity of our paradigm.

\section{General discussion}

In this study, we aimed to investigate whether the choice history bias generalizes to classic intertemporal choice. In a series of three experiments, we created sequences of choices in which one of two options - a soon-small (SS) or a late-large (LL) option — was initially more attractive but became less attractive throughout the sequence. This sequential manipulation based on participants' individual discounting allowed us to measure the choice history bias (Experiment 1-3). Additionally, we manipulated the inter-trial interval (Experiment 3, preregistered). Over this series of experiments, we showed that choice history bias occurs in sequences of intertemporal choices, and that its emergence depends on the task's inter-trial interval.

With these results, our study corroborates previous findings from our lab demonstrating similar effects in a gamified delay discounting task, for which we also found the choice history bias (Scherbaum et al., 2013; Scherbaum et al., 2016; Schoemann \& Scherbaum, 2020) and its modulation by the the inter-trial interval (Senftleben et al., 2020; Senftleben et al., 2019). Importantly, using this gamified discounting task in our previous research, we identified that these biases are neither based on a repetition priming of any option's features nor are they simple effects of residual motor activation/motor priming (Scherbaum et al., 2016). In the task we presented here, we also intended to excluded option or motor priming as an alternative explanation for the choice history bias. To that aim, we implemented the task with rotating choice options. Hence the (relative) position of the options was still predictable, and a mere motor repetition would have acted against the effect we investigated (see Figure 2). However, in the current task, we cannot rule out option priming eliciting the history bias effect and we have to rely on the evidence from our previous work on the history bias using the gamified delay discounting task (Scherbaum et al., 2016). In sum, a choice history bias seems to be present for a range of delay discounting decisions including intertemporal choice. Adding to this, our results are in line with a growing body of research showing that not only perceptual (e.g., Akaishi et al., 2014), but also preferential decisions are influences by previous choices (e.g., Alós-Ferrer et al., 2016; van Rooij et al., 2013). Even in the reinforcement learning literature, the importance of this effect has been acknowledged via a successful 'perseveration' parameter (Wimmer et al., 2012, e.g., ).

The choice history bias points to the path-dependence of intertemporal choices which bears two implications. The first implication is that models of intertemporal choice need to consider the dependency of decisions across trials. While models of intertemporal choice usually focus on describing the general tendency of choice with discounting functions (Doyle, 2013) or the choice process within a trial (Dai \& Busemeyer, 2014; Rodriguez et al., 2014; Scherbaum et al., 2012; Zhao et al., 2019), the choice repetition effects points to the necessity of considering the processes across choices, as they are captured by models that have only recently been applied to delay discounting choices, such as simple attractor models (Scherbaum et al., 2016), or have not yet been applied, such as parallel constrained satisfaction models (cf. Glöckner \& Betsch, 2008; Glöckner et al., 2014). Due to their inherent dynamics that can also act across trials, such models lend themselves to model history biases, as it has been done for perceptual decision making (Berlemont \& Nadal, 2019; Bonaiuto et al., 2016; Hämmerer et al., 2016; Tsetsos et al., 2012).

The second implication is that studying intertemporal choices empirically asks for considering this path-dependence to avoid biased conclusions about individual choices. While a randomization of choices poses no problem in principle in computer-based setups that scan a wide range of option combinations (e.g., Scherbaum et al., 2013), two types of setups are more vulnerable to history biases: first, staircase procedures and second, paper-based measures. In staircase procedures (Berry et al., 2014; O'Hora et al., 2016; Rodzon et al., 2011), each decision moves the participants to a separate subspace of options and hence, a bias on one decision by the previous decision might lead participants one step to far into the wrong decision subspace. In paper-based measures, the use of static decision sequences, for instance, in the Monetary Choice Questionnaire (K. N. Kirby et al., 1999), might also lead to the bias systematically affecting certain decisions. In both cases, one might ask whether this simply leads to a static bias in the estimation across participants and whether this is in the end, important. However, when it comes to the study of differences between populations, the bias might lead to wrong conclusions, since one cannot be sure whether one population discounts more strongly because of increased impulsivity (Green, Fry, et al., 1994; Green et al., 1999; K. N. Kirby \& Maraković, 1996; K. N. Kirby et al., 1999), or because of a decreased history bias. Such considerations show that a choice history bias is not merely a theoretically interesting phenomenon, but could influence the interpretation of findings in the field. 
Despite the mentioned implications, our findings also come with a limitation: From Experiment 1 to Experiment 2, we introduced both a short, controlled ITI and time pressure into our ITC paradigm in order to make it most similar to our gamified delay discounting task. Using the adapted ITC paradigm, we then found the expected choice history bias and concluded that the ITI must have been the driving factor for eliciting the bias effect which eventually led to Experiment 3 . Since the results of Experiment 3 did not fully support our ITI hypotheses, it is worthwhile to consider time pressure as alternative factor for eliciting the bias effect. Previous research in perceptual decision making has shown that the choice history bias is stronger in trials that follow fast previous decisions (Braun et al., 2018; Urai et al., 2017). From this logic it seems plausible that shortening the decision time via time pressure might cause the history bias. However, the same research also showed that fast decision times are associated with high decision confidence (Braun et al., 2018; Urai et al., 2017). Hence, the association between fast previous decisions and the history bias is modulated by decision confidence in the previous trial which suggests an internal rather than an external mechanism being responsible for the history bias. In other words, introducing time pressure does decrease decision times through an external constraint, but might not increase decision confidence which then drives the choice bias in the subsequent trial.

Here, we propose residual activity in the decision system as mechanism driving the history bias. Alternatively, faster evidence accumulation in favour of the previously chosen option has been proposed (Urai et al., 2019). Obviously, without additional assumptions, both accounts do not predict that constraining the decision time through time pressure would alter the choice history bias. However, if the faster evidence accumulation in favour of the previously chosen option was modulated by an initial attention bias (Krajbich et al., 2010; Krajbich \& Rangel, 2011), then time pressure might help the attention bias to take effect and this effect might diminish when decision time is not constrained. Further research is required to shed light on the role of attentional mechanisms during choice repetition.

Taking together, our finding of a choice history bias in intertemporal choice adds to the literature on other biases in intertemporal choice, such as the status-quo bias (Weber et al., 2007), the order of option presentation (Scherbaum et al., 2018b), or the measurement procedure (Hardisty et al., 2013). These biases stand in contrast to the long-held assumption that intertemporal choices reflect an individual's trait-like tendency to discount future rewards (Odum, 2011), which underlies many studies investigating differences between controls and other populations, such as users of alcohol (Mitchell et al., 2005; Petry, 2001), nicotine (Bickel et al., 1999; Lawyer et al., 2011; Ohmura et al., 2005), cocaine (Coffey et al., 2003; Heil et al., 2006; Johnson, 2012), and heroin (Karakula et al., 2016; K. N. Kirby et al., 1999; Madden et al., 1997). However, the malleability of intertemporal choice has recently gained increasing attention (Lempert \& Phelps, 2016) and our study adds to this new perspective, that asks for changes in models and the empirical approach to intertemporal choices—changes that incorporate the established knowledge from perceptual decision making.

\section{Appendix}

\section{Generalized Linear Mixed Modell (GLMM) analysis}

In order to corroborate the ANOVA results reported in the main text, we also examined choice repetition with a Generalized Linear Mixed Model (GLMM) at the trial level, using a logistic link function and the Laplace approximation. The analysis was implemented using the glmer function of the lme4 package for R (Bates et al., 2015). For each experiment, we ran three models: the random-intercept-only model (null model), a model with random intercepts and fixed slopes, and a model with random intercepts and random slopes. Then we ran model comparison and plotted the marginal effects from the winning model using the ggpredict function of the ggeffects package for R (Lüdecke, 2018).

\subsection{Experiment 1}

\subsubsection{Random-intercept-only}

The random-intercept-only model only included a random intercept for each subject and served as null model. The inter-class correlation coefficient (ICC) score was $\mathrm{ICC}_{\mathrm{M} 0}=.24$, the Bayesian information criterion was BIC $=9445.9$ with a $-\mathrm{LL}=-4714.1$.

\subsubsection{Random-intercept-fixed-slopes}

The random-intercept-fixed-slopes model included random intercepts for each subject as well as fixed slopes for the predictors Distance and Direction, as well as for their interaction Distance $\times$ Direction. Only the fixed effect of Distance was significant, $\beta=-10.03, \mathrm{SE}=0.31, z=-32.03, p<.001$. Both the fixed effect of Direction, $\beta=-0.01, \mathrm{SE}=$ $0.06, z=-0.14, p=0.89$, and the interaction Distance $\times$ Direction, $\beta=0.58, \mathrm{SE}=0.41, z=1.41, p=0.16$, did not reach significance. The Bayesian information criterion was $\mathrm{BIC}=6302.5$ with a $-\mathrm{LL}=-3129.1$. 


\subsubsection{Random-intercept-random-slopes}

For the random-intercept-random-slopes model we used the same model as the random-intercept-fixed-slopes model, but we added a random slope for each predictor. Again, only the fixed effect of Distance was significant, $\beta=-13.42, \mathrm{SE}=$ $1.34, z=-10.04, p<.001$. Both the fixed effect of Direction, $\beta=0.09, \mathrm{SE}=0.14, z=0.66, p=0.51$, and the interaction Distance $\times$ Direction, $\beta=1.20, \mathrm{SE}=0.72, z=1.66, p=0.10$, did not reach significance. The Bayesian information criterion was $\mathrm{BIC}=5820.2$ with a $-\mathrm{LL}=-2848.0$.

\subsubsection{Model comparison and marginal effects plot}

Next, we tested if the more complex models actually outperformed the simpler models (i.e., if they explained significantly more variance). The random-intercept-random-slopes model, as the most complex models, outperformed both the null model, $\chi^{2}(3)=3170.04, p<.001$, and the fixed-slopes model, $\chi^{2}(9)=562.07, p<.001$. The same result was suggested by comparison of the BIC values. Hence, and as shown in Figure A8, the random-intercept-random-slopes model corroborated our ANOVA results showing that the probability of choosing the LL option only depends on the factor Distance, and not—as expected—on the factor Direction, too.

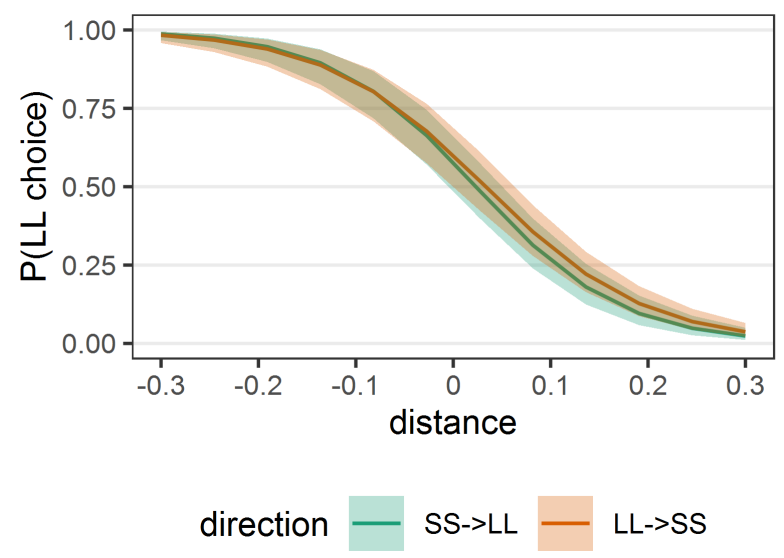

Figure 8: Marginal fixed effects of the random-intercept-random-slopes model fitted to the choice data of Experiment 1. Note: Error areas depict 95\% CIs.

\subsection{Experiment 2}

\subsubsection{Random-intercept-only}

The random-intercept-only model only included a random intercept for each subject and served as null model. The inter-class correlation coefficient (ICC) score was $\mathrm{ICC}_{\mathrm{M} 0}=.34$, the Bayesian information criterion was $\mathrm{BIC}=8559.3$ with a $-\mathrm{LL}=-4270.9$.

\subsubsection{Random-intercept-fixed-slopes}

The random-intercept-fixed-slopes model included random intercepts for each subject as well as fixed slopes for the predictors Distance and Direction, as well as for their interaction Distance $\times$ Direction. The fixed effect of Distance, $\beta=-7.17, \mathrm{SE}=0.26, z=-27.17, p<.001$, and Direction, $\beta=0.52, \mathrm{SE}=0.06, z=8.42, p<.001$, were significant. The interaction Distance $\times$ Direction, $\beta=0.13, \mathrm{SE}=0.36, z=0.35, p=0.73$, did not reach significance. The Bayesian information criterion was $\mathrm{BIC}=6672.0$ with a $-\mathrm{LL}=-3314.0$.

\subsubsection{Random-intercept-random-slopes}

For the random-intercept-random-slopes model we used the same model as the random-intercept-fixed-slopes model, but we added a random slope for each predictor. Again, fixed effects of Distance, $\beta=-7.89, \mathrm{SE}=0.69, z=-11.51, p<$ .001 , and Direction, $\beta=0.54, \mathrm{SE}=0.13, z=4.33, p<.001$, were significant. The interaction Distance $\times$ Direction, $\beta=-0.03, \mathrm{SE}=0.45, z=-0.07, p=0.95$, did not reach significance. The Bayesian information criterion was $\mathrm{BIC}=6498.0$ with a $-\mathrm{LL}=-3187.4$. 


\subsubsection{Model comparison and marginal effects plot}

Next, we tested if the more complex models actually outperformed the simpler models (i.e., if they explained significantly more variance). Again, the random-intercept-random-slopes model, as the most complex models, outperformed both the null model, $\chi^{2}(3)=1913.74, p<.001$, and the fixed-slopes model, $\chi^{2}(9)=253.24, p<.001$. The same result was suggested by comparison of the BIC values. Hence, and as shown in Figure A9, the random-intercept-randomslopes model corroborated our ANOVA results showing that the probability of choosing the LL option depends_-as expected_ — on the factors Distance and Direction.

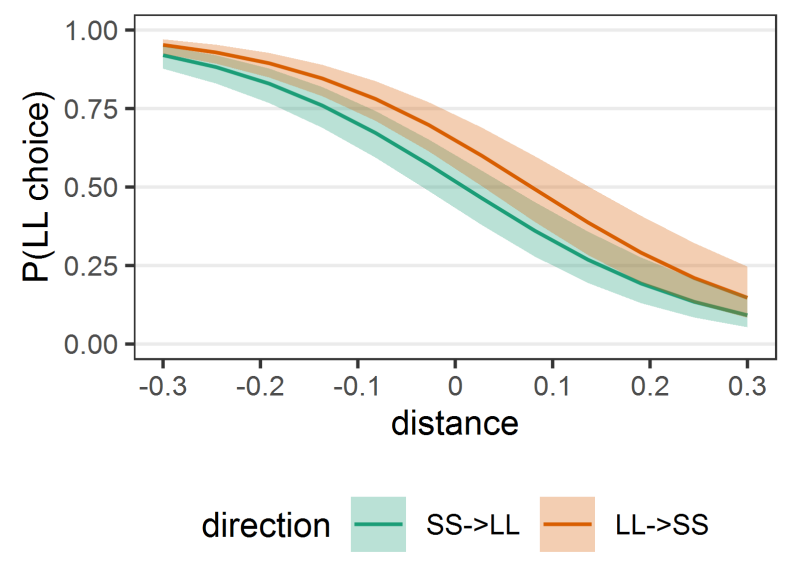

Figure 9: Marginal fixed effects of the random-intercept-random-slopes model fitted to the choice data of Experiment 2. Note: Error areas depict $95 \%$ CIs.

\subsection{Experiment 3}

\subsubsection{Random-intercept-only}

The random-intercept-only model only included a random intercept for each subject and served as null model. The inter-class correlation coefficient (ICC) score was $\mathrm{ICC}_{\mathrm{M} 0}=.44$, the Bayesian information criterion was $\mathrm{BIC}=17784.7$ with a $-\mathrm{LL}=-8882.8$.

\subsubsection{Random-intercept-fixed-slopes}

The random-intercept-fixed-slopes model included random intercepts for each subject as well as fixed slopes for the predictors Distance, Direction and ITI, as well as for their interactions. Please see Table A1 for the results of the fixed effects. The Bayesian information criterion was BIC $=13559.2$ with a $-\mathrm{LL}=-6736.6$.

Table 1: Results of the fixed effects for the random-intercept-fixed-slopes model.

\begin{tabular}{lrrrr}
\hline & $\beta$ & $S E$ & $z$ & $p$ \\
\hline ITI & 0.18 & 0.06 & 2.94 & .003 \\
Direction & 0.20 & 0.06 & 3.36 & .001 \\
Distance & -8.54 & 0.30 & -28.35 & $<.001$ \\
ITI $\times$ Direction & -0.06 & 0.08 & -0.72 & .470 \\
ITI $\times$ Distance & -1.36 & 0.43 & -3.12 & .002 \\
Direction $\times$ Distance & 0.24 & 0.42 & 0.58 & .560 \\
ITI $\times$ Direction $\times$ Distance & 0.77 & 0.61 & 1.27 & .210 \\
\hline
\end{tabular}

\subsubsection{Random-intercept-random-slopes}

For the random-intercept-random-slopes model we used the same model as the random-intercept-fixed-slopes model, but we added a random slope for each predictor. Please see Table A2 for the results of the fixed effects. The Bayesian information criterion was BIC $=12849.7$ with a $-\mathrm{LL}=-6286.4$. 
Table 2: Results of the fixed effects for the random-intercept-random-slopes model.

\begin{tabular}{rllll}
\hline & $\beta$ & $S E$ & $z$ & $p$ \\
\hline ITI & 0.17 & 0.19 & 0.89 & .370 \\
Direction & 0.20 & 0.11 & 1.73 & .080 \\
Distance & -9.98 & 0.90 & -11.18 & $<.001$ \\
ITI $\times$ Direction & -0.08 & 0.15 & -0.56 & .580 \\
ITI $\times$ Distance & -2.25 & 0.51 & -4.38 & $<.001$ \\
Direction $\times$ Distance & 0.55 & 0.54 & 1.03 & .300 \\
ITI $\times$ Direction $\times$ Distance & 1.68 & 0.70 & 2.41 & .020 \\
\hline
\end{tabular}

\subsubsection{Model comparison and marginal effects plot}

Next, we tested if the more complex models actually outperformed the simpler models (i.e., if they explained significantly more variance). Again, the random-intercept-random-slopes model, as the most complex models, outperformed both the null model, $\chi^{2}(7)=4292.37, p<.001$, and the fixed-slopes model, $\chi^{2}(20)=900.45, p<.001$. The same result was suggested by comparison of the BIC values. Hence, and as shown in Figure A10, the random-intercept-random-slopes model corroborated our ANOVA results showing that the probability of choosing the LL option strongly depends on the factor Distance. Additionally, the GLMM analysis revealed the impact of the factor ITI in the form of an interaction with the remaining factors. Here, the three-way-interaction suggested that choice repetition is more likely to occur in trials with short rather than long ITI.

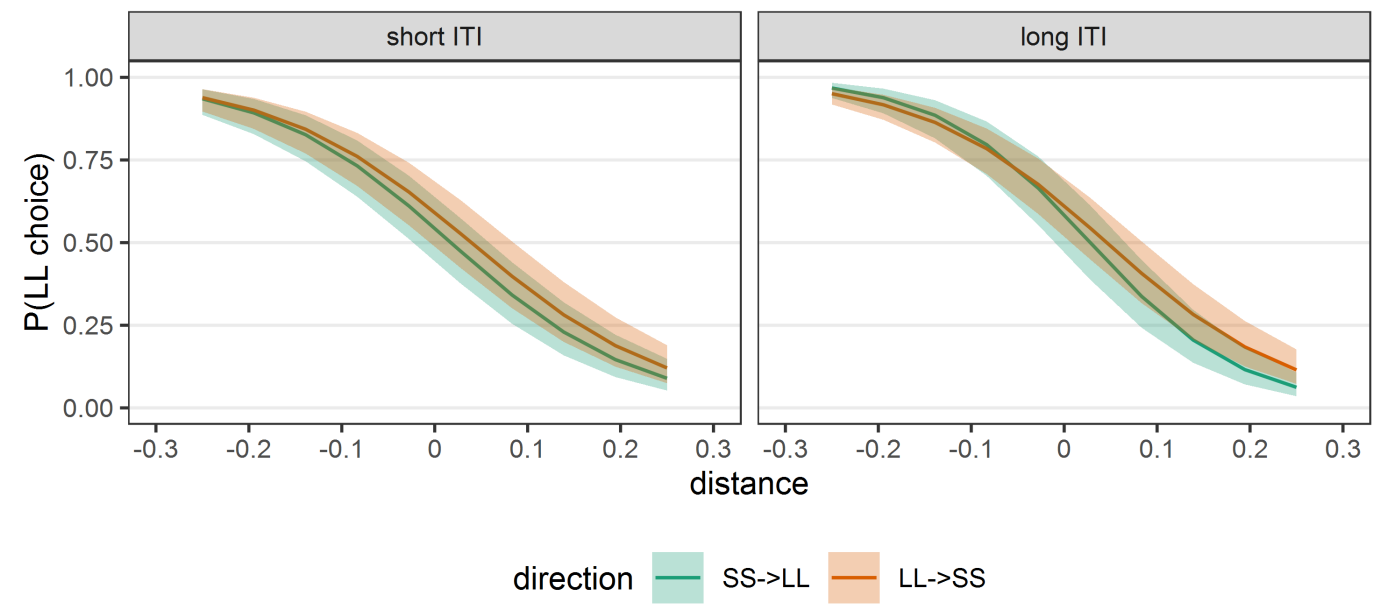

Figure 10: Marginal fixed effects of the random-intercept-random-slopes model fitted to the choice data of Experiment 3. Note: Error areas depict $95 \%$ CIs.

\section{References}

Abrahamyan, A., Silva, L. L., Dakin, S. C., Carandini, M., \& Gardner, J. L. (2016). Adaptable history biases in human perceptual decisions. Proceedings of the National Academy of Sciences, 113(25), E3548-E3557. https://doi.org/10.1073/pnas.1518786113

Akaishi, R., Umeda, K., Nagase, A., \& Sakai, K. (2014). Autonomous mechanism of internal choice estimate underlies decision inertia. Neuron, 81(1), 195-206. https://doi.org/10.1016/j.neuron.2013.10.018

Alós-Ferrer, C., Hügelschäfer, S., \& Li, J. (2016). Inertia and decision making. Frontiers in Psychology, 7. https: //doi.org/10.3389/fpsyg.2016.00169

Baron, J. (2008). Thinking and deciding (4th ed.). Cambridge University Press.

Bates, D., Mächler, M., Bolker, B., \& Walker, S. (2015). Fitting linear mixed-effects models using lme4. Journal of Statistical Software, 67(1), 1-48. https://doi.org/10.18637/jss.v067.i01 
Berlemont, K., \& Nadal, J.-P. (2019). Perceptual decision-making: Biases in post-error reaction times explained by attractor network dynamics. The Journal of Neuroscience, 39(5), 833-853. https://doi.org/10.1523/jneurosci. $1015-18.2018$

Berry, M. S., Sweeney, M. M., Morath, J., Odum, A. L., \& Jordan, K. E. (2014). The nature of impulsivity: Visual exposure to natural environments decreases impulsive decision-making in a delay discounting task. PLoS ONE, 9(5), e97915. https://doi.org/10.1371/journal.pone.0097915

Bertelson, P. (1965). Serial choice reaction-time as a function of response versus signal-and-response repetition. Nature, 206(4980), 217-218. https://doi.org/https://doi.org/10.1038/206217a0

Bickel, W. K., Odum, A. L., \& Madden, G. J. (1999). Impulsivity and cigarette smoking: Delay discounting in current, never, and ex-smokers. Psychopharmacology, 146(4), 447-454. https://doi.org/10.1007/p100005490

Bogacz, R., Usher, M., Zhang, J., \& McClelland, J. L. (2007). Extending a biologically inspired model of choice: Multi-alternatives, nonlinearity and value-based multidimensional choice. Philosophical Transactions of the Royal Society B: Biological Sciences, 362, 1655-1670. https://doi.org/10.1098/rstb.2007.2059

Bogdanov, M., Ruff, C. C., \& Schwabe, L. (2015). Transcranial stimulation over the dorsolateral prefrontal cortex increases the impact of past expenses on decision-making. Cerebral Cortex, bhv298. https://doi.org/10.1093/ cercor/bhv298

Bonaiuto, J. J., de Berker, A., \& Bestmann, S. (2016). Response repetition biases in human perceptual decisions are explained by activity decay in competitive attractor models. eLife, 5. https://doi.org/10.7554/elife.20047

Brainard, D. H. (1997). The psychophysics toolbox. Spatial Vision, 10(4), 433-436. https://doi.org/https://doi.org/10. 1163/156856897X00357

Braun, A., Urai, A. E., \& Donner, T. H. (2018). Adaptive history biases result from confidence-weighted accumulation of past choices. The Journal of Neuroscience, 38(10), 2418-2429. https://doi.org/10.1523/jneurosci.2189-17.2017

Case, P., Tuller, B., Ding, M., \& Kelso, J. A. S. (1995). Evaluation of a dynamical model of speech perception. Perception \& Psychophysics, 57(7), 977-988. https://doi.org/10.3758/bf03205457

Cho, R. Y., Nystrom, L. E., Brown, E. T., Jones, A. D., Braver, T. S., Holmes, P. J., \& Cohen, J. D. (2002). Mechanisms underlying dependencies of performance on stimulus history in a two-alternative forced-choice task. Cognitive, Affective, \& Behavioral Neuroscience, 2(4), 283-299. https://doi.org/10.3758/cabn.2.4.283

Coffey, S. F., Gudleski, G. D., Saladin, M. E., \& Brady, K. T. (2003). Impulsivity and rapid discounting of delayed hypothetical rewards in cocaine-dependent individuals. Experimental and Clinical Psychopharmacology, 11(1), 18-25. https://doi.org/10.1037/1064-1297.11.1.18

Dai, J., \& Busemeyer, J. R. (2014). A probabilistic, dynamic, and attribute-wise model of intertemporal choice. Journal of Experimental Psychology: General, 143(4), 1489-1514. https://doi.org/10.1037/a0035976

Doyle, J. R. (2013). Survey of time preference, delay discounting models. Judgment and Decision Making, 8(2), $116-135$.

Dshemuchadse, M., Scherbaum, S., \& Goschke, T. (2013). How decisions emerge: Action dynamics in intertemporal decision making. Journal of Experimental Psychology: General, 142(1), 93-100. https://doi.org/10.1037/ a0028499

Erev, I., Ert, E., Plonsky, O., Cohen, D., \& Cohen, O. (2017). From anomalies to forecasts: Toward a descriptive model of decisions under risk, under ambiguity, and from experience. Psychological Review, 124(4), 369-409. https://doi.org/10.1037/rev0000062

Faul, F., Erdfelder, E., Buchner, A., \& Lang, A.-G. (2009). Statistical power analyses using g*power3.1 : Testsforcorrelationandregressionanalyses. Behavior Research Methods, 41(4), 1149-1160. https:// doi.org/10.3758/brm.41.4.1149

Franco-Watkins, A. M., Mattson, R. E., \& Jackson, M. D. (2015). Now or later? attentional processing and intertemporal choice. Journal of Behavioral Decision Making, 29(2-3), 206-217. https://doi.org/10.1002/bdm.1895

Frank, T. D., Profeta, V. L. S., \& Harrison, H. S. (2015). Interplay between order-parameter and system parameter dynamics: Considerations on perceptual-cognitive-behavioral mode-mode transitions exhibiting positive and negative hysteresis and on response times. Journal of Biological Physics, 41(3), 257-292. https://doi.org/10. 1007/s10867-015-9378-z

Gao, J., Wong-Lin, K., Holmes, P., Simen, P., \& Cohen, J. D. (2009). Sequential effects in two-choice reaction time tasks: Decomposition and synthesis of mechanisms. Neural Computation, 21(9), 2407-2436. https: //doi.org/10.1162/neco.2009.09-08-866

Glöckner, A., \& Betsch, T. (2008). Modelling option and strategy choices with connectionist networks: Towards an intergrative model of automatic and deliberate decision making. Judgment and Decision Making, 3(3), 215-228.

Glöckner, A., Hilbig, B. E., \& Jekel, M. (2014). What is adaptive about adaptive decision making? a parallel constraint satisfaction account. Cognition, 133(3), 641-666. https://doi.org/10.1016/j.cognition.2014.08.017

Gold, J. I., \& Shadlen, M. N. (2007). The neural basis of decision making. Annual Review of Neuroscience, 30(1), 535-574. https://doi.org/10.1146/annurev.neuro.29.051605.113038 
Green, L., Fristoe, N., \& Myerson, J. (1994). Temporal discounting and preference reversals in choice between delayed outcomes. Psychonomic Bulletin \& Review, 1(3), 383-389. https://doi.org/10.3758/bf03213979

Green, L., Fry, A. F., \& Myerson, J. (1994). Discounting of delayed rewards: A life-span comparison. Psychological Science, 5(1), 33-36. https://doi.org/10.1111/j.1467-9280.1994.tb00610.x

Green, L., Myerson, J., \& Ostaszewski, P. (1999). Discounting of delayed rewards across the life span: Age differences in individual discounting functions. Behavioural Processes, 46(1), 89-96. https://doi.org/10.1016/s03766357(99)00021-2

Greiner, B. (2015). Subject pool recruitment procedures: Organizing experiments with ORSEE. Journal of the Economic Science Association, 1(1), 114-125. https://doi.org/10.1007/s40881-015-0004-4

Hale, D. J. (1967). Sequential effects in a two-choice serial reaction task. Quarterly Journal of Experimental Psychology, 19(2), 133-141. https://doi.org/10.1080/14640746708400082

Haller, A., \& Schwabe, L. (2014). Sunk costs in the human brain. NeuroImage, 97, 127-133. https://doi.org/10.1016/j. neuroimage.2014.04.036

Hämmerer, D., Bonaiuto, J., Klein-Flügge, M., Bikson, M., \& Bestmann, S. (2016). Selective alteration of human value decisions with medial frontal tDCS is predicted by changes in attractor dynamics. Scientific Reports, $6(1)$. https://doi.org/10.1038/srep25160

Hardisty, D. J., Thompson, K. F., Krantz, D. H., \& Weber, E. U. (2013). How to measure time preferences: An experimental comparison of three methods. Judgment \& Decision Making, 8(3).

Heil, S. H., Johnson, M. W., Higgins, S. T., \& Bickel, W. K. (2006). Delay discounting in currently using and currently abstinent cocaine-dependent outpatients and non-drug-using matched controls. Addictive Behaviors, 31(7), 1290-1294. https://doi.org/10.1016/j.addbeh.2005.09.005

Hilbert, M. (2012). Toward a synthesis of cognitive biases: How noisy information processing can bias human decision making. Psychological Bulletin, 138(2), 211-237. https://doi.org/10.1037/a0025940

Hock, H. S., Schöner, G., \& Giese, M. (2003). The dynamical foundations of motion pattern formation: Stability, selective adaptation, and perceptual continuity. Perception \& Psychophysics, 65(3), 429-457. https://doi.org/ $10.3758 / \mathrm{bf03194574}$

Hunt, L. T. (2014). What are the neural origins of choice variability? Trends in Cognitive Sciences, 18(5), 222-224. https://doi.org/10.1016/j.tics.2014.01.004

Hunt, L. T., Kolling, N., Soltani, A., Woolrich, M. W., Rushworth, M. F. S., \& Behrens, T. E. J. (2012). Mechanisms underlying cortical activity during value-guided choice. Nature Neuroscience, 15(3), 470-476. https://doi.org/ 10.1038/nn.3017

Jocham, G., Hunt, L. T., Near, J., \& Behrens, T. E. J. (2012). A mechanism for value-guided choice based on the excitation-inhibition balance in prefrontal cortex. Nature Neuroscience, 15(7), 960-961. https://doi.org/10. 1038/nn.3140

Johnson, M. W. (2012). An efficient operant choice procedure for assessing delay discounting in humans: Initial validation in cocaine-dependent and control individuals. Experimental and Clinical Psychopharmacology, 20(3), 191-204. https://doi.org/10.1037/a0027088

Johnson, M. W., \& Bickel, W. K. (2002). Within-subject comparison of real and hypothetical money rewards in delay discounting. Journal of the Experimental Analysis of Behavior, 77(2), 129-146. https://doi.org/10.1901/jeab. 2002.77-129

Karakula, S. L., Weiss, R. D., Griffin, M. L., Borges, A. M., Bailey, A. J., \& McHugh, R. (2016). Delay discounting in opioid use disorder: Differences between heroin and prescription opioid users. Drug and Alcohol Dependence, 169, 68-72. https://doi.org/10.1016/j.drugalcdep.2016.10.009

Kelso, J. A. S. (1997). Dynamic pattern: The self-organization of brain and behavior. MIT Press.

Kelso, J. A. S., Case, P., Holroyd, T., Horvath, E., Rączaszek, J., Tuller, B., \& Ding, M. (1995). Multistability and metastability in perceptual and brain dynamics, 159-184. https://doi.org/10.1007/978-3-642-78411-8_9

Kirby, K. N., \& Maraković, N. N. (1996). Delay-discounting probabilistic rewards: Rates decrease as amounts increase. Psychonomic Bulletin \& Review, 3(1), 100-104. https://doi.org/10.3758/bf03210748

Kirby, K. N., Petry, N. M., \& Bickel, W. K. (1999). Heroin addicts have higher discount rates for delayed rewards than non-drug-using controls. Journal of Experimental Psychology: General, 128(1), 78-87.

Kirby, N. H. (1976). Sequential effects in two-choice reaction time: Automatic facilitation or subjective expectancy? Journal of Experimental Psychology: Human Perception and Performance, 2(4), 567-577. https://doi.org/10. 1037/0096-1523.2.4.567

Kleinschmidt, A., Büchel, C., Hutton, C., Friston, K. J., \& Frackowiak, R. S. J. (2002). The neural structures expressing perceptual hysteresis in visual letter recognition. Neuron, 34(4), 659-666. https://doi.org/10.1016/s08966273(02)00694-3

Krajbich, I., Armel, C., \& Rangel, A. (2010). Visual fixations and the computation and comparison of value in simple choice. Nature Neuroscience, 13(10), 1292-1298. https://doi.org/10.1038/nn.2635 
Krajbich, I., \& Rangel, A. (2011). Multialternative drift-diffusion model predicts the relationship between visual fixations and choice in value-based decisions. Proceedings of the National Academy of Sciences, 108(33), 13852-13857. https://doi.org/10.1073/pnas.1101328108

Lawyer, S. R., Schoepflin, F., Green, R., \& Jenks, C. (2011). Discounting of hypothetical and potentially real outcomes in nicotine-dependent and nondependent samples. Experimental and Clinical Psychopharmacology, 19(4), 263-274. https://doi.org/10.1037/a0024141

Lempert, K. M., \& Phelps, E. A. (2016). The malleability of intertemporal choice. Trends in Cognitive Sciences, 20(1), 64-74. https://doi.org/10.1016/j.tics.2015.09.005

Lüdecke, D. (2018). ggeffects: Tidy data frames of marginal effects from regression models. Journal of Open Source Software, 3(26), 772. https://doi.org/10.21105/joss.00772

Madden, G. J., Petry, N. M., Badger, G. J., \& Bickel, W. K. (1997). Impulsive and self-control choices in opioiddependent patients and non-drug-using control patients: Drug and monetary rewards. Experimental and Clinical Psychopharmacology, 5(3), 256-262. https://doi.org/10.1037/1064-1297.5.3.256

Mitchell, J. M., Fields, H. L., D'Esposito, M., \& Boettiger, C. A. (2005). Impulsive responding in alcoholics. Alcoholism: Clinical and Experimental Research, 29(12), 2158-2169. https://doi.org/10.1097/01.alc.0000191755.63639.4a

Myerson, J., Green, L., \& Warusawitharana, M. (2001). Area under the curve as a measure of discounting. Journal of the Experimental Analysis of Behavior, 76(2), 235-243. https://doi.org/10.1901/jeab.2001.76-235

Nicolle, A., Fleming, S. M., Bach, D. R., Driver, J., \& Dolan, R. J. (2011). A regret-induced status quo bias. Journal of Neuroscience, 31(9), 3320-3327. https://doi.org/10.1523/jneurosci.5615-10.2011

Odum, A. L. (2011). Delay discounting: Trait variable? Behavioural Processes, 87(1), 1-9. https://doi.org/10.1016/j. beproc.2011.02.007

Ohmura, Y., Takahashi, T., \& Kitamura, N. (2005). Discounting delayed and probabilistic monetary gains and losses by smokers of cigarettes. Psychopharmacology, 182(4), 508-515. https://doi.org/10.1007/s00213-005-0110-8

O’Hora, D., Carey, R., Kervick, A., Crowley, D., \& Dabrowski, M. (2016). Decisions in motion: Decision dynamics during intertemporal choice reflect subjective evaluation of delayed rewards. Scientific Reports, 6(1). https: //doi.org/10.1038/srep20740

Pelli, D. G. (1997). The VideoToolbox software for visual psychophysics: Transforming numbers into movies. Spatial Vision, 10(4), 437-442. https://doi.org/10.1163/156856897X00366

Perea, M., \& Carreiras, M. (2003). Sequential effects in the lexical decision task: The role of the item frequency of the previous trial. The Quarterly Journal of Experimental Psychology: Section A, 56(3), 385-401. https: //doi.org/10.1080/02724980244000387

Petry, N. M. (2001). Delay discounting of money and alcohol in actively using alcoholics, currently abstinent alcoholics, and controls. Psychopharmacology, 154(3), 243-250. https://doi.org/10.1007/s002130000638

Quinlan, P. T. (1999). Sequential effects in auditory choice reaction time tasks. Psychonomic Bulletin \& Review, 6(2), 297-303. https://doi.org/10.3758/bf03212333

Remington, R. J. (1969). Analysis of sequential effects on choice reaction times. Journal of Experimental Psychology, 82(2), 250-257. https://doi.org/10.1037/h0028122

Rodriguez, C. A., Turner, B. M., \& McClure, S. M. (2014). Intertemporal choice as discounted value accumulation. PLoS ONE, 9(2), e90138. https://doi.org/10.1371/journal.pone.0090138

Rodzon, K., Berry, M. S., \& Odum, A. L. (2011). Within-subject comparison of degree of delay discounting using titrating and fixed sequence procedures. Behavioural Processes, 86(1), 164-167. https://doi.org/10.1016/j. beproc.2010.09.007

Scherbaum, S., Dshemuchadse, M., \& Goschke, T. (2012). Building a bridge into the future: Dynamic connectionist modeling as an integrative tool for research on intertemporal choice. Frontiers in Psychology, 3. https: //doi.org/10.3389/fpsyg.2012.00514

Scherbaum, S., Dshemuchadse, M., Leiberg, S., \& Goschke, T. (2013). Harder than expected: Increased conflict in clearly disadvantageous delayed choices in a computer game. PLoS ONE, 8(11), e79310. https://doi.org/10. 1371/journal.pone.0079310

Scherbaum, S., Frisch, S., \& Dshemuchadse, M. (2018a). A bird in the hand isn't good for long. Experimental Psychology, 65(1), 23-31. https://doi.org/10.1027/1618-3169/a000385

Scherbaum, S., Frisch, S., \& Dshemuchadse, M. (2018b). Step by step: Harvesting the dynamics of delay discounting decisions. Quarterly Journal of Experimental Psychology, 71(4), 949-964. https://doi.org/10.1080/17470218. 2017.1307863

Scherbaum, S., Frisch, S., Leiberg, S., Lade, S. J., Goschke, T., \& Dshemuchadse, M. (2016). Process dynamics in delay discounting decisions: An attractor dynamics approach. Judgement and Decision Making, 11(5), 472-495.

Schoemann, M., \& Scherbaum, S. (2020). From high- to one-dimensional dynamics of decision making: Testing simplifications in attractor models. Cognitive Processing, 21(2), 303-313. https://doi.org/10.1007/s10339-02000953-z 
Sekuler, R. (1996). Motion perception: A modern view of wertheimer's 1912 monograph. Perception, 25(10), 12431258. https://doi.org/10.1068/p251243

Senftleben, U., Schoemann, M., Rudolf, M., \& Scherbaum, S. (2020). To stay or not to stay: The stability-flexibility trade-off in value-based decision making. Quarterly Journal of Experimental Psychology. https://doi.org/10. $1177 / 1747021820964330$

Senftleben, U., Schoemann, M., Schwenke, D., Richter, S., Dshemuchadse, M., \& Scherbaum, S. (2019). Choice perseveration in value-based decision making: The impact of inter-trial interval and mood. Acta Psychologica, 198, 102876. https://doi.org/10.1016/j.actpsy.2019.102876

Soetens, E., Melis, A., \& Notebaert, W. (2004). Sequence learning and sequential effects. Psychological Research, 69(1-2), 124-137. https://doi.org/10.1007/s00426-003-0163-4

Soetens, E. (1998). Localizing sequential effects in serial choice reaction time with the information reduction procedure. Journal of Experimental Psychology: Human Perception and Performance, 24(2), 547-568. https://doi.org/10. 1037/0096-1523.24.2.547

Soetens, E., Boer, L. C., \& Hueting, J. E. (1985). Expectancy or automatic facilitation? Separating sequential effects in two-choice reaction time. Journal of Experimental Psychology: Human Perception and Performance, 11(5), 598-616. https://doi.org/10.1037/0096-1523.11.5.598

Tsetsos, K., Gao, J., McClelland, J. L., \& Usher, M. (2012). Using time-varying evidence to test models of decision dynamics: Bounded diffusion vs. the leaky competing accumulator model. Frontiers in Neuroscience, 6 . https://doi.org/10.3389/fnins.2012.00079

Tuller, B., Case, P., Ding, M., \& Kelso, J. A. S. (1994). The nonlinear dynamics of speech categorization. Journal of Experimental Psychology: Human Perception and Performance, 20(1), 3-16. https://doi.org/10.1037/00961523.20.1.3

Tversky, A., \& Kahneman, D. (1974). Judgment under uncertainty: Heuristics and biases. Science, 185(4157), 11241131. https://doi.org/10.1126/science.185.4157.1124

Urai, A. E., Braun, A., \& Donner, T. H. (2017). Pupil-linked arousal is driven by decision uncertainty and alters serial choice bias. Nature Communications, 8(1), 14637. https://doi.org/10.1038/ncomms 14637

Urai, A. E., de Gee, J. W., Tsetsos, K., \& Donner, T. H. (2019). Choice history biases subsequent evidence accumulation. eLife, 8. https://doi.org/10.7554/elife.46331

Urminski, O., \& Zauberman, G. (2014). The wiley blackwell handbook of judgment and decision making. In G. Keren \& G. Wu (Eds.). Wiley. https://doi.org/10.1002/9781118468333

Usher, M., \& McClelland, J. L. (2001). The time course of perceptual choice: The leaky, competing accumulator model. Psychological Review, 108(3), 550-592. https://doi.org/10.1037/0033-295x.108.3.550

Usher, M., \& McClelland, J. L. (2004). Loss aversion and inhibition in dynamical models of multialternative choice. Psychological Review, 111(3), 757-769. https://doi.org/10.1037/0033-295x.111.3.757

van Rooij, M. M. J. W., Favela, L. H., Malone, M., \& Richardson, M. J. (2013). Modeling the dynamics of risky choice. Ecological Psychology, 25(3), 293-303. https://doi.org/10.1080/10407413.2013.810502

Vervaeck, K. R., \& Boer, L. C. (1980). Sequential effects in two-choice reaction time: Subjective expectancy and automatic after- effect at short response-stimulus intervals. Acta Psychologica, 44(2), 175-190. https://doi.org/ 10.1016/0001-6918(80)90066-9

Wang, X.-J. (2008). Decision making in recurrent neuronal circuits. Neuron, 60(2), 215-234. https://doi.org/10.1016/j. neuron.2008.09.034

Wang, X.-J. (2012). Neural dynamics and circuit mechanisms of decision-making. Current Opinion in Neurobiology, 22(6), 1039-1046. https://doi.org/10.1016/j.conb.2012.08.006

Weber, E. U., Johnson, E. J., Milch, K. F., Chang, H., Brodscholl, J. C., \& Goldstein, D. G. (2007). Asymmetric discounting in intertemporal choice. Psychological Science, 18(6), 516-523. https://doi.org/10.1111/j.14679280.2007.01932.x

Wimmer, G. E., Daw, N. D., \& Shohamy, D. (2012). Generalization of value in reinforcement learning by humans. European Journal of Neuroscience, 35(7), 1092-1104. https://doi.org/10.1111/j.1460-9568.2012.08017.x

Wong, K.-F., \& Wang, X.-J. (2006). A recurrent network mechanism of time integration in perceptual decisions. Journal of Neuroscience, 26(4), 1314-1328. https://doi.org/10.1523/jneurosci.3733-05.2006

Wong, K.-F., Huk, A. C., Shadlen, M. N., \& Wang, X.-J. (2007). Neural circuit dynamics underlying accumulation of time-varying evidence during perceptual decision making. Frontiers in Computational Neuroscience, 1, 1-12. https://doi.org/10.3389/neuro.10.006.2007

Wulff, D. U., \& van den Bos, W. (2017). Modeling choices in delay discounting. Psychological Science, 29(11), 1890-1894. https://doi.org/10.1177/0956797616664342

Zhao, W. J., Diederich, A., Trueblood, J. S., \& Bhatia, S. (2019). Automatic biases in intertemporal choice. Psychonomic Bulletin \& Review, 26(2), 661-668. https://doi.org/10.3758/s13423-019-01579-9 